\title{
The surgery exact sequence, K-theory and the signature operator
}

\author{
Paolo Piazza and Thomas Schick
}

September 22, 2018

\begin{abstract}
The main result of this paper is a new and direct proof of the natural transformation from the surgery exact sequence in topology to the analytic K-theory sequence of Higson and Roe.

Our approach makes crucial use of analytic properties and new index theorems for the signature operator on Galois coverings with boundary. These are of independent interest and form the second main theme of the paper. The main technical novelty is the use of large scale index theory for Dirac type operators that are perturbed by lower order operators.
\end{abstract}

\section{Contents}

\section{Introduction}

2 Index and rho classes defined by perturbations 4

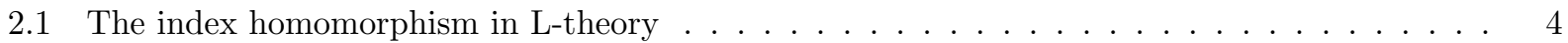

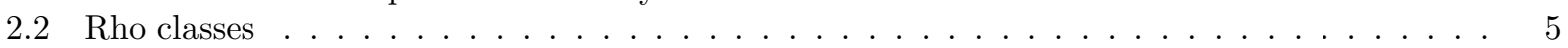

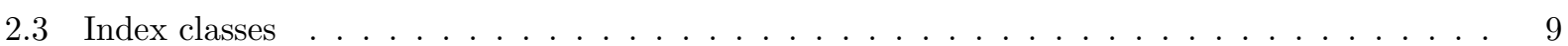

3 Delocalized APS-index theorem for perturbed operators 13

4 Mapping the surgery sequence to K-Theory

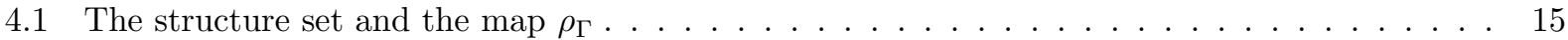

4.2 The set $\mathcal{N}(V)$ and the $\operatorname{map} \beta_{\Gamma}: \mathcal{N}(V) \rightarrow K_{n}(B \Gamma) \ldots \ldots \ldots \ldots \ldots \ldots$

4.3 Mapping the surgery sequence to the Higson-Roe sequence . . . . . . . . . . . . . . . . . 17

5 Proof of the delocalized APS index theorem $\mathbf{2 0}$

5.1 Reduction to the cylinder . . . . . . . . . . . . . . . . . . . . . . . . . 20

5.2 Proof of the cylinder delocalized index theorem for perturbed operators . . . . . . . . . . 222

\section{Introduction}

Let $V$ be a smooth, closed, oriented $n$-dimensional manifold. We consider $\Gamma:=\pi_{1}(V)$ and the universal cover $\widetilde{V} \rightarrow V$. Finally, we let $\tilde{u}: \widetilde{V} \rightarrow E \Gamma$ be a $\Gamma$-equivariant map covering a classifying map $u: V \rightarrow B \Gamma$ for $\widetilde{V} \rightarrow V$. Associated to this data there are two important exact sequences.

The first one, due to Browder, Novikov, Sullivan and Wall, is the surgery exact sequence in topology [26, 17, 12:

$$
\cdots \rightarrow L_{n+1}(\mathbb{Z} \Gamma) \rightarrow \mathcal{S}(V) \rightarrow \mathcal{N}(V) \rightarrow L_{n}(\mathbb{Z} \Gamma) .
$$

The central object of interest in this sequence is the structure set $\mathcal{S}(V)$; elements in the set $\mathcal{S}(V)$ are given by homotopy manifold structures on $V$, i.e. orientation preserving homotopy equivalences $f: M \rightarrow V$, with $M$ a smooth oriented closed manifold, considered up to $h$-cobordism. $\mathcal{N}(V)$ is the set of degree one normal 
maps $f: M \rightarrow V$ considered up to normal bordism. Finally, the abelian groups $L_{*}(\mathbb{Z} \Gamma)$, the $L$-groups of the integral group ring $\mathbb{Z} \Gamma$, are defined algebraically but have a geometric realization as cobordism groups of manifolds with boundary with additional structure on the boundary.

The surgery exact sequence 1.1 plays a fundamental role in the classification of high-dimensional smooth compact manifolds.

The second exact sequence associated to $\widetilde{V} \rightarrow V$ is purely analytic and is due to Higson and Roe. Consider the $C^{*}$-algebra $C^{*}(\widetilde{V})^{\Gamma}$ called the Roe algebra and obtained as the closure of the $\Gamma$-equivariant locally compact finite propagation operators; this is an ideal in $D^{*}(\widetilde{V})^{\Gamma}$, the $C^{*}$-algebra obtained as the closure of the $\Gamma$-equivariant pseudolocal finite propagation operators. There is a short exact sequence of $C^{*}$-algebras

$$
0 \rightarrow C^{*}(\tilde{V})^{\Gamma} \rightarrow D^{*}(\widetilde{V})^{\Gamma} \rightarrow D^{*}(\widetilde{V})^{\Gamma} / C^{*}(\tilde{V})^{\Gamma} \rightarrow 0
$$

and thus a 6-terms long exact sequence in K-theory

$$
\cdots \rightarrow K_{n+1}\left(C^{*}(\widetilde{V})^{\Gamma}\right) \rightarrow K_{n+1}\left(D^{*}(\widetilde{V})^{\Gamma}\right) \rightarrow K_{n+1}\left(D^{*}(\widetilde{V})^{\Gamma} / C^{*}(\widetilde{V})^{\Gamma}\right) \stackrel{\partial}{\rightarrow} K_{n}\left(C^{*}(\tilde{V})^{\Gamma}\right) \rightarrow \cdots
$$

There are canonical isomorphisms,

$$
K_{*+1}\left(D^{*}(\widetilde{V})^{\Gamma} / C^{*}(\widetilde{V})^{\Gamma}\right)=K_{*}(V) \quad \text { and } \quad K_{*}\left(C^{*}(\widetilde{V})^{\Gamma}\right)=K_{*}\left(C_{r}^{*} \Gamma\right)
$$

with $C_{r}^{*} \Gamma$ denoting, as usual, the reduced $C^{*}$-algebra of the group $\Gamma$. Thus we can rewrite the long exact sequence in K-theory as

$$
\cdots \rightarrow K_{n+1}\left(C_{r}^{*} \Gamma\right) \rightarrow K_{n+1}\left(D^{*}(\tilde{V})^{\Gamma}\right) \rightarrow K_{n}(V) \rightarrow K_{n}\left(C_{r}^{*} \Gamma\right) \rightarrow \cdots
$$

This is the analytic surgery sequence of Higson and Roe.

By [18, the map $K_{*}(V) \rightarrow K_{*}\left(C_{r}^{*} \Gamma\right)$ is precisely the (Baum-Connes) assembly map. This connects the Higson-Roe surgery sequence to fundamental questions such as the Strong Novikov Conjecture or the Baum-Connes conjecture.

In a series of papers [2, 3, 4, Higson and Roe constructed the following remarkable commutative diagram:

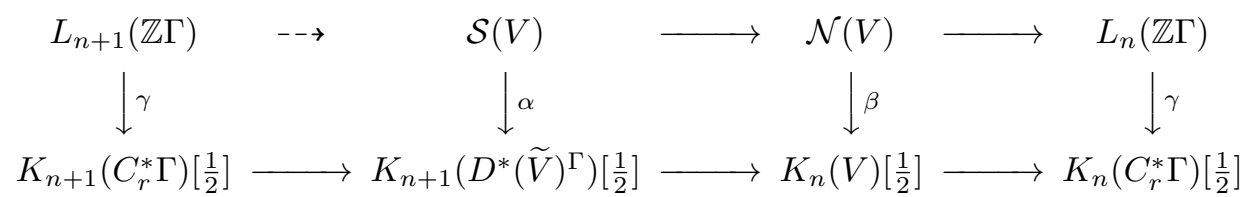

where $A\left[\frac{1}{2}\right]$ is a shorthand for $A \otimes_{\mathbb{Z}} \mathbb{Z}\left[\frac{1}{2}\right]$ if $A$ is any abelian group.

It is important to mention that both $\alpha$ and $\gamma$ in $\sqrt{1.2}$ are constructed using fine properties of Poincaré spaces that are not smooth manifolds. The main ingredient is the use of homotopy equivalences to glue manifolds with boundary along their boundaries. The resulting objects are not manifolds, but still Poincaré complexes. These have well defined (higher) signatures which then feature in the construction.

One main goal of this article is to give an alternative and direct route to the transformation from the smooth surgery exact sequence in topology to the analytic surgery sequence of Higson-Roe. The main point of our construction is that we use index theoretic constructions, applied to the signature operator, throughout.

Our approach follows the one presented in our recent paper [16], where we showed how to map the exact sequence of Stolz for positive scalar curvature metrics to the Higson-Roe exact sequence. Throughout, we will follow the notations of our paper [16, the current paper should be considered as a companion to the latter one, with the signature operator replacing the Dirac operator in a fundamental way.

Our main result, Theorem 4.10 , is that there are natural (index theoretic) $\operatorname{maps} \operatorname{Ind}_{\Gamma}, \rho, \beta$

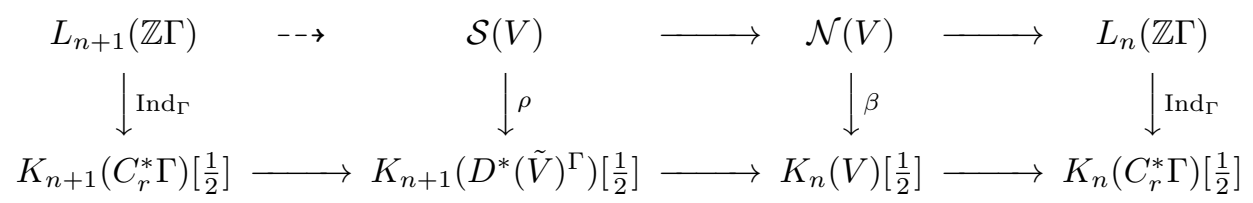


making the diagram commutative.

The main technical novelty, compared to the companion paper [16, is that we have to use an orientation preserving homotopy $f: M \rightarrow V$ to perturb the signature operator (on the disjoint union of $M$ and $V$ ) to an invertible operator. We follow an explicit recipe for such a perturbation $C_{f}$ initiated by Hilsum-Skandalis in their fundamental work [5], and then modified by Piazza and Schick in [15] and further modified by Wahl in 25. We will use the latter version of this perturbation and for this reason we name it Wahl's perturbation. One advantage of choosing Wahl's perturbation is that it makes it possible to extend most of the results of Hilsum and Skandalis from closed manifolds to manifolds with boundary. See [25, Theorem 8.4].

Notice that the perturbed operator looses several of the appealing properties of a Dirac type operator (like unit propagation of the associated wave operator). Therefore, the second main theme of this work is the proof of a new coarse index theorem for the signature operator on Galois coverings with boundary. This is of independent interest. The main technical novelty is the use of large scale index theory for Dirac type operators that are perturbed by lower order operators.

More explicitly, in (1.4) the map $\rho$ is defined applying the following idea: if $M \stackrel{f}{\rightarrow} V$ is a homotopy equivalence then $\rho[M \stackrel{f}{\rightarrow} V]$ is defined in terms of the projection onto the positive part of the spectrum of the self-adjoint invertible operator $D+C_{f}$, with $D$ the signature operator on the Galois covering defined by $(f \circ u) \sqcup(-u): M \sqcup(-V) \rightarrow B \Gamma$ and $C_{f}$ is Wahl's perturbation defined by $f$.

A geometrically given cycle for $L_{n}(\mathbb{Z} \Gamma)$ consists in particular of a manifold with boundary (made up of two components), and the extra datum of a homotopy equivalence of the boundaries of the pieces. Wahl's perturbation for this homotopy equivalence can then be used to perturb the signature operator to be invertible at the boundary. This allows for the definition of a generalized Atiyah-Patodi-Singer index class $\operatorname{Ind}(D, f)$ in $K_{n+1}\left(C_{r}^{*} \Gamma\right)$, which in the end defines the map $\operatorname{Ind}_{\Gamma}$.

Finally, $\beta$ is defined as in Higson and Roe: if $f: M \rightarrow V$ defines a class in $\mathcal{N}(V)$ then its image through $\beta$ is obtained as $f_{*}\left(\left[D_{M}\right]\right)-\left[D_{V}\right] \in K_{n}(V)$, with $\left[D_{M}\right]$ and $\left[D_{V}\right]$ the fundamental classes associated to the signature operators on the smooth compact manifolds $M$ and $V$.

We shall first treat the case in which $V$ is odd dimensional and only at the end indicate how the results of this paper and of [16] can also be extended to the even dimensional case.

It should be added that the map $\operatorname{Ind}_{\Gamma}$ of our diagram, the one out of $L_{*}(\mathbb{Z} \Gamma)$, has already been constructed by Charlotte Wahl in 25]. We will make use of her important results for parts of our program. The main novelty in the approach presented in this paper and in [25], compared to [2, 3, 4, is therefore the definition of the map $\operatorname{Ind}_{\Gamma}$, the definition of $\rho$ and, crucially, the proof of well-definedness and of commutativity of the squares.

All this we prove by establishing and then employing a delocalized Atiyah-Patodi-Singer index theorem for perturbed Dirac operators:

1.5 Theorem (Theorem 3.1). Let $W$ be an oriented manifold with free cocompact orientation preserving action and with boundary $M_{1} \sqcup M_{2}$. Let $f: M_{1} \rightarrow M_{2}$ be an orientation preserving $\Gamma$-equivariant homotopy equivalence. Then

$$
\iota_{*}(\operatorname{Ind}(D, f))=j_{*}\left(\rho\left(D_{\partial}+C_{f}\right)\right) \quad \text { in } \quad K_{0}\left(D^{*}(\widetilde{W})^{\Gamma}\right) .
$$

Here, $D$ is the signature operator on $W, \operatorname{Ind}(D, f)$ is the generalized APS index class associated to $D$ and to the homotopy equivalence $f, j: D^{*}(\partial \widetilde{W})^{\Gamma} \rightarrow D^{*}(\widetilde{W})^{\Gamma}$ is the homomorphism induced by the inclusion $\partial \widetilde{W} \rightarrow \widetilde{W}$ and $\iota: C^{*}(\widetilde{W})^{\Gamma} \rightarrow D^{*}(\widetilde{W})^{\Gamma}$ the inclusion.

It should be added that this result generalizes to general Dirac type operators with more abstract boundary perturbations making the boundary operator invertible. See Theorem 3.1 for a precise statement.

The main novelty in Theorem 4.10 i.e. the construction and the commutativity of the diagram (1.4), compared to the analogous result in [16, is the treatment of the technicalities which arise when dealing with perturbed Dirac type operators. 
1.6 Remark. By using the signature operator of Hilsum and Teleman it is possible to extend our results to Lipschitz manifolds and thus, by Sullivan's theorem, to the surgery exact sequence in the category TOP of topological manifolds. We refer the reader to Zenobi's 31] where stability results for the topological structure set under products are also discussed.

1.7 Remark. Because of their fundamental result, Higson and Roe name the group $K_{0}\left(D_{\Gamma}^{*}\right)$ the analytic structure group; they denote it $\mathcal{S}_{1}(\Gamma)$. Similarly, the analytic structure group $\mathcal{S}_{0}(\Gamma)$ is, by definition, the group $K_{1}\left(D_{\Gamma}^{*}\right)$. In [1], Deeley and Goffeng have introduced a geometric structure group $\mathcal{S}_{1}^{\text {geo }}(\Gamma)$, with cycles defined à la Baum-Douglas. Using the results of the present paper, Deeley and Goffeng have proved that their geometric definition is isomorphic to the analytic one given by Higson and Roe.

Acknowledgments. We are glad to thank Georges Skandalis, Stephane Vassout, Charlotte Wahl, Rudolf Zeidler and Vito Felice Zenobi for interesting discussions. We thank the anonymous referee for a very careful reading of the original manuscript and for valuable suggestions. P.P. thanks the Projet Algèbres d'Opérateurs of Institut de Mathématiques de Jussieu for hospitality and financial support while part of this research was carried out; he also thanks Mathematisches Institut and the Courant Research Center "Higher order structures in mathematics" for their hospitality for several visits to Göttingen; the financial support of Ministero dell'Università e della Ricerca Scientifica (through the project "Spazi di Moduli e Teoria di Lie") is also gratefully acknowledged. T.S. acknowledges the support of the Courant Research Center "Higher order structures in mathematics".

\section{Index and rho classes defined by perturbations}

\subsection{The index homomorphism in L-theory}

The map $\operatorname{Ind}_{\Gamma}: L_{n+1}(\mathbb{Z} \Gamma) \rightarrow K_{n+1}\left(C_{r}^{*} \Gamma\right)$ has been defined by Wahl, building on results of Hilsum-Skandalis [5] and the authors [15]. We briefly describe it. Assume that $n+1$ is even. Recall, see for example [4. Chapter 4] and the references therein, that an element $x \in L_{n+1}(\mathbb{Z} \Gamma)$ is represented by a quadruple $(W, F, X \times[0,1], u: X \rightarrow B \Gamma)$ with $W$ a cobordism between two smooth orientable manifolds $\partial_{1} W$ and $\partial_{2} W$, $X$ a smooth orientable manifold, $F:(W, \partial W) \rightarrow(X \times[0,1], \partial(X \times[0,1]))$ a degree one normal map of pairs, $f_{1}:=\left.F\right|_{\partial_{1} W}$ and $f_{2}:=\left.F\right|_{\partial_{2} W}$ oriented homotopy equivalences and $u: X \rightarrow B \Gamma$ a classifying map. Let $f=f_{1} \sqcup f_{2}$ denote the restriction of $F$ to $\partial W$. Consider $Z:=W \sqcup X \times[0,1]$, a manifold with boundary. Let $\mathcal{D}_{Z}$ be the signature operator on $Z$ with coefficients in the Mishchenko bundle defined by $u: X \rightarrow B \Gamma$ and $u \circ F: W \rightarrow B \Gamma$, i.e. the bundle obtained as pullback of the $C_{r}^{*} \Gamma$-module bundle $E \Gamma \times{ }_{\Gamma} C_{r}^{*} \Gamma$ over $B \Gamma$. Then, proceeding as in [25], we can construct a smoothing perturbation $\mathcal{C}_{f}$ of the boundary operator $\mathcal{D}_{\partial Z}$ with the property that $\mathcal{D}_{\partial Z}+\mathcal{C}_{f}$ is invertible. This perturbation, a smoothing version of the original one defined by Hilsum and Skandalis in [5], is, first of all, a self-adjoint bounded operator on the $C_{r}^{*} \Gamma$-Hilbert module $\mathcal{E}_{\mathrm{M}}:=L^{2}\left(Z, \Lambda^{*} Z \otimes \mathcal{F}_{\mathrm{M}}\right)$ with $\mathcal{F}_{\mathrm{M}}$ denoting the Mishchenko bundle. Moreover, it is an element in $\Psi_{C_{r}^{*} \Gamma}^{-\infty}\left(Z, \Lambda^{*} Z \otimes \mathcal{F}_{\mathrm{M}}\right)$, the smoothing operators in the Mishchenko-Fomenko calculus. Wahl's perturbation $\mathcal{C}_{f}$ is an example of what is called, in the literature, a smoothing trivializing perturbation; more generally a trivializing perturbation for $\mathcal{D}$ is a self-adjoint $C_{r}^{*} \Gamma$-bounded operator $\mathcal{C}$ with the property that $\mathcal{D}+\mathcal{C}$ is invertible as an unbounded operator on the Hilbert module $\mathcal{E}_{\mathrm{M}}$. The original perturbation defined by Hilsum-Skandalis is an example of such a perturbation, see [31, Proposition 3.1] for a proof. Notice that in this paper only the smoothing trivializing perturbation $\mathcal{C}_{f}$ will be used; this corresponds to the choice $\epsilon \in(0,+\infty)$ in Wahl's treatment.

We extend Wahl's perturbation $\mathcal{C}_{f}$ in the obvious way to the cylinder $\mathbb{R} \times \partial Z$ (we extend it to be constant in the cylindrical direction) and then use a cut-off function in order to graft this operator to the manifold with cylindrical end $Z_{\infty}$ associated to $Z$. We denote this global perturbation by $\mathcal{C}_{f, \infty}$; this is the global perturbation chosen by Wahl and it is the one we shall take. (In previous work on higher APS index theory the global perturbation $\mathcal{C}_{f, \infty}$ was chosen to be $b$-pseudodifferential, see [13] [8]; while this choice would simplify some of our arguments in Section 2.3 , it would eventually make the proof of our main theorem more involved; 
this is why we have chosen the perturbation just explained.) Proceeding as in [8, and [7, Theorem 10.1] one proves that there is a well defined index class associated to $\mathcal{D}_{\infty}+\mathcal{C}_{f, \infty}$, with $\mathcal{D}$ denoting the MishchenkoFomenko signature operator. The index class is an element in $K_{n+1}\left(C_{r}^{*} \Gamma\right)$. See Section 2.3.2 below for further details. Thus, to the quadruple $(W, F, X \times[0,1], u: X \rightarrow B \Gamma)$ we associate $\operatorname{Ind}\left(\mathcal{D}_{\infty}+\mathcal{C}_{f, \infty}\right) \in K_{n+1}\left(C_{r}^{*} \Gamma\right)$.

2.1 Theorem. This construction induces a well defined group homomorphism

$$
\operatorname{Ind}_{\Gamma}: L_{n+1}(\mathbb{Z} \Gamma) \rightarrow K_{n+1}\left(C_{r}^{*} \Gamma\right)
$$

Proof. This is proved by Charlotte Wahl in [25, Theorem 9.1].

We give more information on this index class in Section 2.3 .

\subsection{Rho classes}

In this subsection we first fix a $\Gamma$-manifold $\widetilde{V}$ with a free cocompact action of $\Gamma$ with quotient $V$, a smooth compact manifold without boundary. We fix a $\Gamma$-invariant metric on $\widetilde{V}$; we also fix a $\Gamma$-equivariant hermitian vector bundle $\widetilde{E}$ on $\widetilde{V}$ with quotient $E$ on $V$. We assume the existence of a $\Gamma$-equivariant Clifford structure on $\widetilde{E}$ and we denote by $D$ the corresponding Dirac type operator on $\widetilde{V}$; this is a $\Gamma$-equivariant operator. Notice that we do not employ the tilde-notation for the operators on the covering. We denote by $\mathcal{D}$ the induced operator in the Mishchenko-Fomenko calculus.

Recall the main players in the Higson-Roe surgery sequence. We have the $C^{*}$-algebra $C^{*}(\tilde{V})^{\Gamma}$, called Roe algebra, obtained as the closure of the locally compact $\Gamma$-invariant finite propagation operators, and we have the $C^{*}$-algebra $D^{*}(\tilde{V})^{\Gamma}$, obtained as the closure of $\Gamma$-invariant pseudolocal finite propagation operators. Recall also that $C^{*}(\tilde{V})^{\Gamma}$ is an ideal in $D^{*}(\tilde{V})^{\Gamma}$. We refer to the companion paper [16] for the precise definitions and for the notation we adopt. One of the extra subtleties is that one has to stabilize the bundles by tensoring with $l^{2}(\mathbb{N})$ for the definition in particular of $D^{*}(\tilde{V})^{\Gamma}$ and let operators act on one corner $V \otimes \mathbb{C} e_{1} \subset V \otimes l^{2}(\mathbb{N})$. See for example [16, Section 1.2]. We will suppress this throughout in the notation.

\subsubsection{Operators on the covering}

Recall from [16] that given a cocompact Galois covering $\widetilde{V} \rightarrow V$ there is an isomorphism

$$
\mathbb{K}\left(\mathcal{E}_{\mathrm{M}}\right) \cong C^{*}(\widetilde{V})^{\Gamma}
$$

where we recall that $\mathcal{E}_{\mathrm{M}}$ stands for the Mishchenko $C_{r}^{*} \Gamma$-Hilbert module $L^{2}\left(V, E \otimes \mathcal{F}_{\mathrm{M}}\right)$, with $\mathcal{F}_{\mathrm{M}}=\widetilde{V} \times_{\Gamma} C_{r}^{*} \Gamma$, the Mishchenko bundle. Consider now $V$ and $\widetilde{V}$ as above. Let $\mathcal{C} \in \Psi_{C_{r}^{*} \Gamma}^{-\infty}\left(V, E \otimes \mathcal{F}_{\mathrm{M}}\right)$ be a smoothing trivializing perturbation for a Dirac type operator $\mathcal{D} \in \operatorname{Diff}_{C_{r}^{*} \Gamma}^{1}\left(V, E \otimes \mathcal{F}_{\mathrm{M}}\right)$. Obviously, from the MishchenkoFomenko calculus, we have that $\Psi_{C_{r}^{*} \Gamma}^{-\infty}\left(V, E \otimes \mathcal{F}_{\mathrm{M}}\right) \subset \mathbb{K}\left(\mathcal{E}_{\mathrm{M}}\right)$. Using $(2.2)$ we obtain immediately that $\mathcal{C}$ defines an element $C$ in $C^{*}(\widetilde{V})^{\Gamma}$.

In what follows we have two goals in mind: on the one hand we wish to generalize the assignment of the operator $C$ to any self-adjoint bounded operator $\mathcal{C}$ (not necessarily a smoothing operator in the MishchenkoFomenko calculus); on the other hand we wish to give a precise definition for such a $C$. To this end let $\pi: C_{r}^{*} \Gamma \rightarrow \mathcal{B}\left(\ell^{2}(\Gamma)\right)$ be the left regular representation. Recall e.g. from [20] that tensoring with $\pi$ (a faithful representation) induces an isomorphism of right Hilbert $\Gamma$-modules

$$
\mathcal{E}_{\mathrm{M}} \otimes_{\pi} \ell^{2}(\Gamma) \rightarrow L^{2}\left(\tilde{V}, \Lambda^{*} \widetilde{V}\right) .
$$

Associating to each $\mathcal{C} \in \mathbb{B}\left(\mathcal{E}_{\mathrm{M}}\right)$ the operator $\mathcal{C} \otimes_{\pi} \operatorname{Id}_{\ell^{2}(\Gamma)}$ in $\mathcal{B}\left(\mathcal{E}_{\mathrm{M}} \otimes_{\pi} \ell^{2}(\Gamma)\right)$ we define a homomorphism of $C^{*}$-algebras

$$
\mathbb{B}\left(\mathcal{E}_{\mathrm{M}}\right) \ni \mathcal{C} \mapsto \mathcal{C} \otimes_{\pi} \operatorname{Id}_{\ell^{2}(\Gamma)} \in \mathcal{B}\left(\mathcal{E}_{\mathrm{M}} \otimes_{\pi} \ell^{2}(\Gamma)\right) .
$$


Notice that the right hand side is $\Gamma$-equivariant, given that $\mathcal{C}$ is $C_{r}^{*} \Gamma$-linear. Thus, conjugating with 2.3), we obtain a $C^{*}$-homomorphism

$$
L_{\pi}: \mathbb{B}\left(\mathcal{E}_{\mathrm{M}}\right) \ni \mathcal{C} \mapsto C_{\pi} \in \mathcal{B}\left(L^{2}\left(\widetilde{V}, \Lambda^{*} \widetilde{V}\right)\right)^{\Gamma}
$$

2.4 Proposition. $L_{\pi}$ sends $\mathbb{K}\left(\mathcal{E}_{\mathrm{M}}\right)$ isomorphically onto $C^{*}(\tilde{V})^{\Gamma}$. Moreover, it sends $\mathbb{B}\left(\mathcal{E}_{\mathrm{M}}\right)$ into $\mathfrak{M}\left(C^{*}(\tilde{V})^{\Gamma}\right)$, the multiplier algebra of $C^{*}(\tilde{V})^{\Gamma}$.

Proof. $L_{\pi}$ is injective, given that $\pi$ is faithful. The statement about $L_{\pi}\left(\mathbb{K}\left(\mathcal{E}_{\mathrm{M}}\right)\right)$ follows by looking at the image through $L_{\pi}$ of a dense set in $\mathbb{K}\left(\mathcal{E}_{\mathrm{M}}\right)$. We can choose, for example, $\Psi_{\mathbb{C} \Gamma}^{-\infty}$. If $A \in \Psi_{\mathbb{C} \Gamma}^{-\infty}$ then $L_{\pi}(A)$ is nothing but the associated $\Gamma$-compactly supported smoothing operator on the covering (see 11 , Proposition $6]$ ) and these operators are dense in $C^{*}(\tilde{V})^{\Gamma}$. Thus $L_{\pi}$ sends $\mathbb{K}\left(\mathcal{E}_{\mathrm{M}}\right)$ isomorphically onto $C^{*}(\tilde{V})^{\Gamma}$. The second statement is classical: indeed, Kasparov has proved, see [27, that $\mathbb{B}\left(\mathcal{E}_{\mathrm{M}}\right)$ is the multiplier algebra of $\mathbb{K}\left(\mathcal{E}_{\mathrm{M}}\right)$. The statement now follows from general arguments; indeed, given an injective representation $\phi$ of a $C_{\tilde{\phi}}^{*}$-algebra $K$ into the bounded operators of a Hilbert space $H$, then $\phi$ extends uniquely to a representation $\tilde{\phi}$ of the multiplier algebra of $K$ and $\tilde{\phi}(\mathfrak{M}(K))=\mathfrak{M}(\phi(K))$.

We are now ready to define precisely the bounded operator $C$, on the covering $\tilde{V}$, corresponding to a self-adjoint operator $\mathcal{C} \in \mathbb{B}\left(\mathcal{E}_{\mathrm{M}}\right)$. We simply set

$$
C:=L_{\pi}(\mathcal{C})
$$

We know that this is an element in $\mathfrak{M}\left(C^{*}(\tilde{V})^{\Gamma}\right)$, the multiplier algebra of $C^{*}(\tilde{V})^{\Gamma}$. If $\mathcal{C}$ is, in addition, smoothing, i.e. $\mathcal{C} \in \Psi_{C_{r}^{*} \Gamma}^{-\infty}\left(V, E \otimes \mathcal{F}_{\mathrm{M}}\right)$, then $C \in C^{*}(\tilde{V})^{\Gamma}$.

2.6 Remark. If we apply all this to the smoothing trivializing perturbation $\mathcal{C}_{f}$ defined by a homotopy equivalence $f: M \rightarrow V$, we immediately see that $\mathcal{C}_{f}$ defines a perturbation $C_{f}:=L_{\pi}\left(\mathcal{C}_{f}\right)$ in $C^{*}(\widetilde{Z})^{\Gamma}$, with $Z=M \cup(-V)$ and $\widetilde{Z}$ the Galois covering defined by $f \circ u \cup(-u): M \cup(-V) \rightarrow B \Gamma$, $u$ denoting a classifying map for the universal cover of $V$.

\subsubsection{The rho-class associated to a perturbation}

Let $\mathcal{D} \in \operatorname{Diff}_{C_{r}^{*} \Gamma}^{1}\left(V, E \otimes \mathcal{F}_{\mathrm{M}}\right)$ be a Dirac type operator as above. We first assume $V$ and therefore $\widetilde{V}$ to be odd dimensional. Assume that there exists a trivializing perturbation $\mathcal{C}$ for $\mathcal{D}$. We recall that this means that $\mathcal{C}$ is a self-adjoint bounded operator on $\mathcal{E}_{\mathrm{M}}$, the Mishchenko $C_{r}^{*} \Gamma$-Hilbert module, with the property that the self-adjoint regular operator $\mathcal{D}+\mathcal{C}$ is invertible. Following [13], it is proved in [10] that this is true if and only if the index class $\operatorname{Ind}(\mathcal{D}) \in K_{1}\left(C_{r}^{*} \Gamma\right)$ vanishes.

For the proof of the following proposition see, e.g., the proof of [7, Lemma 2.1].

2.7 Proposition. Let $D$ be the Dirac operator on $\widetilde{V}$ corresponding to $\mathcal{D}$. Let $C:=L_{\pi}(\mathcal{C})$. Then $D+C$ is self-adjoint and $L^{2}$-invertible.

Next we recall a result of Higson-Roe, implicitly proved in [2, Proposition 5.9].

2.8 Proposition. Let $D$ be a self-adjoint unbounded operator on $H:=L^{2}(\widetilde{V}, \widetilde{E})$, as above. Let $\mathfrak{A}$ be a $C^{*}$-algebra in $\mathcal{B}(H)$ and let $\mathfrak{J}$ be an ideal in $\mathfrak{A}$. Let $\mathfrak{M}$ be the multiplier algebra of $\mathfrak{J}$. Assume that $S$ is a self-adjoint operator in $\mathfrak{M}$, that the resolvent of $D$ is in $\mathfrak{J}$ and that $D\left(1+D^{2}\right)^{-1 / 2}$ is in $\mathfrak{A}$. Then the resolvent of $D+S$ is in $\mathfrak{J}$ and $(D+S)\left(1+(D+S)^{2}\right)^{-1 / 2} \in \mathfrak{A}$. Consequently,

- $f(D+S) \in \mathfrak{J}, \quad \forall f \in C_{0}(\mathbb{R})$

- $f(D+S) \in \mathfrak{A}, \quad \forall f \in C([-\infty, \infty])$. 
2.9 Proposition. Let $\mathcal{D}$ and $D$ as above; let $\mathcal{C}$ be a trivializing perturbation for $\mathcal{D}$ and let $C:=L_{\pi}(\mathcal{C}) \in$ $\mathfrak{M}\left(C^{*}(\widetilde{V})^{\Gamma}\right)$ be the corresponding perturbation of $D$ on the covering. Then

$$
\frac{D+C}{|D+C|} \text { is an element in } D^{*}(\widetilde{V})^{\Gamma} \text {. }
$$

Proof. Choose $\mathfrak{A}=D^{*}(\widetilde{V})^{\Gamma}$ and $\mathfrak{J}=C^{*}(\widetilde{V})^{\Gamma}$. Then we can apply Proposition 2.8 given that $C \in$ $\mathfrak{M}\left(C^{*}(\widetilde{V})^{\Gamma}\right)$ (see Proposition 2.4). We thus obtain that $\chi(D+C)$ is an element in $D^{*}(\widetilde{V})^{\Gamma}$ for any chopping function $\chi$; choosing $\chi$ equal to \pm 1 on the spectrum of $D+C$ we are done.

This brings us to the definition of rho-classes.

2.10 Definition. Let $\widetilde{V} \rightarrow V$ a $\Gamma$-covering of a smooth compact orientable odd dimensional manifold $V$ without boundary. Let $D$ be a $\Gamma$-equivariant Dirac operator on $\widetilde{V}$ acting on the sections of a $\Gamma$-equivariant bundle $\widetilde{E}$. Let $\mathfrak{M}$ be the multiplier algebra of $C^{*}(\widetilde{V})^{\Gamma}$. For any self-adjoint operator $A \in \mathfrak{M}$ with the property that $D+A$ is $L^{2}$-invertible we can consider the operator $(D+A) /|D+A|$. Then, proceeding as above we have that

$$
\frac{D+A}{|D+A|} \text { is an element in } D^{*}(\widetilde{V})^{\Gamma} \text {. }
$$

The rho-class associated to $D$ and to the trivializing perturbation $A$ is, by definition, the idempotent defined by the involution $(D+A) /|(D+A)|$ :

$$
\rho(D+A):=\left[\frac{1}{2}\left(\frac{D+A}{|D+A|}+1\right)\right] \in K_{0}\left(D^{*}(\widetilde{V})^{\Gamma}\right) .
$$

In the even dimensional case, with a $\mathbb{Z} / 2$-graded bundle $\widetilde{E}=\widetilde{E}^{+} \oplus \widetilde{E}^{-}$we can proceed analogously, once we have a $\mathbb{Z} / 2$-graded trivializing self-adjoint perturbation $A \in \mathfrak{M}\left(C^{*}(\widetilde{V})^{\Gamma}\right)$, viz.

$$
D+A=\left(\begin{array}{cc}
0 & D^{-}+A^{-} \\
D^{+}+A^{+} & 0
\end{array}\right)
$$

A necessary and sufficient condition for such a perturbation to exist is that $\operatorname{Ind}(\mathcal{D})=0$ in $K_{0}\left(C_{r}^{*} \Gamma\right)$, see [14, 10. In order to define the rho class associated to 2.12 we consider the space, call it $J$, of $\Gamma$-equivariant bundle isometries from $\widetilde{E}^{-}$to $\widetilde{E}^{+}$(more precisely, on their stabilizations, obtained by tensoring the two bundles with $l^{2}(\mathbb{N})$, see the beginning of Section 2.2 this makes sure that the bundles are trivial). Any element $u$ in $J$ induces in a natural way an isometry $U: L^{2}\left(\widetilde{V}, \widetilde{E}^{-}\right) \rightarrow L^{2}\left(\widetilde{V}, \widetilde{E}^{+}\right)$. It is obvious that $U$ commutes with the action of $\Gamma$ and that it covers the identity in the $D^{*}$ sense, see for example [16, Section 1]. Thus we can set

$$
\rho(D+A):=\left[U \chi(D+A)_{+}\right] \in K_{1}\left(D^{*}(\widetilde{V})^{\Gamma}\right)
$$

with $\chi$ an odd chopping function equal to the sign function on the spectrum of $D+A$. Since $J$ is isomorphic, once we stabilize, to the space of $U(H)$-valued functions on the quotient $V=\widetilde{V} / \Gamma$ ( $H$ a separable Hilbert space), and since $U(H)$ is contractible, we see immediately that also $J$ is contractible. Thus, by homotopy invariance of K-theory, the right hand side in (2.13) does not depend on the choice of $u$ in $J$.

2.14 Remark. In our previous paper [16] we allowed for an arbitrary $\Gamma$-equivariant isometry $U: L^{2}\left(\widetilde{V}, \widetilde{E}^{-}\right) \rightarrow$ $L^{2}\left(\widetilde{V}, \widetilde{E}^{+}\right)$covering the identity in the $D^{*}$-sense, see the discussion leading to Definition 1.11. This is not correct for we could then change at will the class on the right hand side of (2.13) by composing on the left with an arbitrary $\Gamma$-equivariant unitary $V \in U\left(L^{2}\left(\widetilde{V}, \widetilde{E}^{-}\right)\right)$, covering the identity in the $D^{*}$-sense. Using the more specific choice of $U$ as above, everything in [16] goes through as stated there.

Finally, if we consider the canonical map $u: \widetilde{V} \rightarrow E \Gamma$ then we define

$$
\rho_{\Gamma}(D+A):=u_{*} \rho(D+A) \in K_{*}\left(D_{\Gamma}^{*}\right) .
$$


2.16 Remark. The rho classes do depend, in general, on the choice of the trivializing perturbation $A$. This will be clear from our delocalized APS index theorem 3.1 for perturbed operators.

2.17 Remark. Note that $K_{*}\left(D_{\Gamma}^{*}\right)$, by the Baum-Connes conjecture, is expected to vanish for torsion-free groups, but is often non-zero otherwise. Therefore, the universal $\rho$-class $\rho_{\Gamma}$ is of interest essentially only if $\Gamma$ is a group with non-trivial torsion.

\subsubsection{Fundamental examples of rho classes}

We present two fundamental examples of $\rho$-classes. As we shall see later these examples enjoy strong stability properties with respect to the trivializing perturbation.

2.18 Definition. Let $(V, g)$ be an oriented smooth Riemannian manifold without boundary with fundamental group $\Gamma$. Let $u: V \rightarrow B \Gamma$ be the classifying map for the universal cover of $V$. Let $(M, h)$ be another oriented Riemannian manifold without boundary and assume that $M \stackrel{f}{\rightarrow} V$ is an oriented homotopy equivalence. We consider $Z=M \sqcup(-V)$ with the obvious classifying map $u_{Z}: Z \rightarrow B \Gamma$ induced by $u$ and by $u \circ f$. We then obtain

$$
\rho\left(D+C_{f}\right) \in K_{n+1}\left(D^{*}(\widetilde{Z})^{\Gamma}\right) \quad \text { and } \quad \rho_{\Gamma}\left(D+C_{f}\right) \in K_{n+1}\left(D_{\Gamma}^{*}\right)
$$

with $D$ equal to the signature operator on the covering $\widetilde{Z}:=u_{Z}^{*} E \Gamma$ and $C_{f}$ the smoothing trivializing perturbation defined by the homotopy equivalence $f$. We choose Wahl's version of this perturbation. Notice that there is an obvious $\Gamma$-equivariant map $\tilde{\phi}: \widetilde{Z} \rightarrow \widetilde{V}, \tilde{\phi}:=\tilde{f} \cup \operatorname{id}_{(-\widetilde{V})}$. We set

$$
\rho(f):=\tilde{\phi}_{*} \rho\left(D+C_{f}\right) \in K_{n+1}\left(D^{*}(\widetilde{V})^{\Gamma}\right) ; \quad \rho_{\Gamma}(f):=\rho_{\Gamma}\left(D+C_{f}\right) \in K_{n+1}\left(D_{\Gamma}^{*}\right) .
$$

Observe that, by functoriality, $\rho_{\Gamma}(f)=\tilde{u}_{*} \rho(f)$.

Proof. We have to argue why $\rho(f)$ and $\rho_{\Gamma}(f)$ do not depend on the choices involved. The signature operator on $Z$ depends on the choice of the Riemannian metrics on $M$ and $V$. The perturbation $\mathcal{C}_{f}$ depends on several choices (see [25] for details); it also depends on the choice of the metric on $M$ and $V$. Wahl proves (compare 25, Section 4]) that two different choices can be joined by a path of invertible operators $D(t)+B(t)$; thus, according to (an easy extension of) Proposition 2.34 below, the rho-class $\rho\left(D+C_{f}\right) \in K_{n+1}\left(D^{*}(\widetilde{Z})^{\Gamma}\right)$ is independent of the choice of the Riemannian metrics and of the choices we have made in defining the trivializing perturbation $C_{f}$. Consequently, also $\rho(f)$ and $\rho_{\Gamma}(f)$ are independent of these choices.

2.20 Example. Let $(M, g)$ be a closed oriented Riemannian manifold and $\widetilde{M} \rightarrow M$ a $\Gamma$-cover. Let $2 m$ or $2 m+1$ be the dimension of $M$, depending whether $M$ is even or odd dimensional. We assume that the Laplacian on differential forms on the covering is $L^{2}$-invertible in degree $m$. We shall say briefly that the Laplacian on the covering is invertible in middle degree. By the homotopy invariance of $L^{2}$-Betti numbers and of the Novikov-Shubin invariants this is a homotopy invariant condition. Let $D$ be the signature operator on $\widetilde{M}$ and let $\mathcal{D}$ be the associated Mishchenko-Fomenko operator As explained in 9] there is then a class of smoothing trivializing perturbations $\mathcal{S}$, that were named symmetric there, and that enjoy strong stability properties, as we shall explain in a moment. We define the rho-class of a manifold $M$ satisfying the above condition as the $K_{*}\left(D^{*}(\widetilde{M})^{\Gamma}\right)$-class

$$
\rho(D+\mathcal{S}) \quad \text { with } \quad \mathcal{S} \text { a symmetric trivializing perturbation. }
$$

with $*=\operatorname{dim} M+1$. We also define $\rho_{\Gamma}(D+\mathcal{S})=\tilde{u}_{*}(\rho(D+\mathcal{S})) \in K_{*}\left(D_{\Gamma}^{*}\right)$. We show in Remark 3.8 that

$$
\rho_{\Gamma}(D+\mathcal{S})=\rho_{\Gamma}\left(D+\mathcal{S}^{\prime}\right) \in K_{*}\left(D_{\Gamma}^{*}\right) \quad \text { if } \mathcal{S}, \mathcal{S}^{\prime} \text { are symmetric trivializing perturbations. }
$$


This brings us to the following definition:

Let $\widetilde{M} \rightarrow M$ a $\Gamma$-cover and assume that the Laplacian on differential forms on $\widetilde{M}$ is invertible in middle degree. Let $D$ be the signature operator on $\widetilde{M}$. We then define $\rho_{\Gamma}(\widetilde{M}) \in K_{*}\left(D_{\Gamma}^{*}\right)$ as

$$
\rho_{\Gamma}(\widetilde{M}):=\rho_{\Gamma}(D+\mathcal{S}) \text { for any symmetric trivializing perturbation } \mathcal{S} .
$$

The notation is justified because, as in the previous example, the right hand side is also independent of the choice of metric. Working a bit harder one can extend these results to odd dimensional Galois coverings $\widetilde{M} \rightarrow M, \operatorname{dim} M=2 m+1$, with the property that in degree $m$ the reduced and unreduced cohomology with values in the local system defined by the Mishchenko-Fomenko bundle are equal. See [7, 23].

\section{$2.3 \quad$ Index classes}

The index map $\operatorname{Ind}_{\Gamma}: L_{n+1}(\mathbb{Z} \Gamma) \rightarrow K_{n+1}\left(C_{r}^{*} \Gamma\right)$ is constructed using APS higher index theory in the Mishchenko-Fomenko framework. We wish to frame the construction of APS index classes on manifolds with boundary and with cylindrical ends in coarse index theory.

\subsubsection{Coarse index classes on manifolds with cylindrical ends}

We will now consider manifolds with boundary and with cylindrical ends. First we recall the basic notation.

2.22 Notation. Let $(W, g)$ be a Riemannian manifold with boundary $M:=\partial W$. We shall always assume $g$ to have product structure near the boundary. We denote by $W_{\infty}$ the manifold $W$ with an infinite semicylinder $[0, \infty) \times \partial M$ attached to the boundary. If $\widetilde{W}$ is a $\Gamma$-covering of $W$, then we similarly denote by $\widetilde{W}_{\infty}$ the manifold obtained from $\widetilde{W}$ by attaching an infinite semi-cylinder.

We will denote by $P_{0}$ the multiplication operator on $\widetilde{W}_{\infty}$ defined by the characteristic function of the subset $[0, \infty) \times \partial \widetilde{W}$ in $\widetilde{W}_{\infty}$; similarly, we denote by $P_{R}$ the multiplication operator defined by the characteristic function of the subset $[R, \infty) \times \partial \widetilde{W}$ in $\widetilde{W}$. We have similar operators defined in $\mathbb{R} \times \partial \widetilde{W}$ and with a small abuse of notation we employ the same symbols.

We assume $W$ to be even dimensional.

We consider a $\Gamma$-equivariant Dirac type operator $D$ on $\widetilde{W}$, acting on the sections of a $\Gamma$-equivariant $\mathbb{Z} / 2$ graded Hermitian vector bundle $\widetilde{E}$. We denote the boundary operator by $D_{\partial}$ and the natural extension of $D$ to $\widetilde{W}_{\infty}$ by $D_{\infty}$. Notice once again that we do not employ the tilde-notation for the operators on the covering.

We denote with $\mathcal{D}, \mathcal{D}_{\partial}$ and $\mathcal{D}_{\infty}$ the corresponding operators in the Mishchenko-Fomenko calculus. We adopt the Clifford and grading conventions of [25].

We consider $C_{\partial}$, a norm limit of $\Gamma$-equivariant self-adjoint bounded finite propagation operators on $L^{2}(\partial \widetilde{W})$ such that $D_{\partial}+C_{\partial}$ is $L^{2}$-invertible. For example $C_{\partial}$ is equal to $L_{\pi}\left(\mathcal{C}_{\partial}\right)$, with $\mathcal{C}_{\partial}$ a smoothing trivializing perturbation for $\mathcal{D}_{\partial}$. We now define a global perturbation on $\widetilde{W}_{\infty}$.

2.23 Definition. We extend $C_{\partial}$ on $\mathbb{R} \times \partial \widetilde{W}$ to be constant in the $\mathbb{R}$ direction. We fix an inward collar on $\widetilde{W}$ diffeomorphic to $[-1,0] \times \widetilde{W}$; we consider $\widetilde{W}_{\infty}$; then using a cutoff function equal to 1 on $[0, \infty)$ and equal to 0 at -1 , we graft this operator to a bounded operator on $\widetilde{W}_{\infty}$, denoted $C_{\infty}^{+}$, acting from the sections of $\widetilde{E}^{+}$to the sections of $\widetilde{E}^{-}$. We set $C_{\infty}^{-}:=\left(C_{\infty}^{+}\right)^{*}$, the formal adjoint of $C_{\infty}^{+}$, and we consider

$$
C_{\infty}:=\left(\begin{array}{cc}
0 & C_{\infty}^{-} \\
C_{\infty}^{+} & 0
\end{array}\right)
$$

We consider the unbounded operator $D_{\infty}+C_{\infty}$, which is odd with respect to the $\mathbb{Z} / 2$-grading induced by $\widetilde{E}$. The operator $C_{\partial}$ is, by assumption, a norm limit of finite propagation operators. Therefore $C_{\infty}$ also has this property. 
More generally, let $B_{\infty}$ be a bounded self-adjoint odd operator on $W_{\infty}$ with the following two properties:

$B_{\infty}$ is norm limit of finite propagation $\Gamma$-operators and $P_{0} B_{\infty} P_{0}-P_{0} C_{\infty} P_{0} \in C^{*}\left(\tilde{W} \subset \tilde{W}_{\infty}\right)^{\Gamma}$.

Notice that $C_{\infty}$ has these properties.

2.25 Lemma. The operators $B_{\infty}$ belongs to the multiplier algebra $\mathfrak{M}\left(C^{*}\left(\widetilde{W}_{\infty}\right)^{\Gamma}\right)$.

Proof. Indeed, as is well known, every operator which is norm limit of $\Gamma$-equivariant finite propagation operators is a multiplier of $C^{*}\left(\widetilde{W}_{\infty}\right)^{\Gamma}$. We give for completness the easy argument: if $A$ is such an operator with propagation $R$ and if $\phi$ is a compactly supported function, then $\phi A=\phi A \psi$ for every compactly supported $\psi$ which is equal to 1 on the $R$-neighborhood of the support of $\phi$. Consequently, if $E \in C^{*}\left(W_{\infty}\right)^{\Gamma}$, $\phi A E=\phi A \psi E$ is compact given that $\psi E$ is compact. Passing to norm limits, the general statement follows.

We can now apply the Higson-Roe result, as stated in Proposition 2.8 , with $\mathfrak{A}=D^{*}\left(\widetilde{W}_{\infty}\right)^{\Gamma}, \mathfrak{J}=C^{*}\left(\widetilde{W}_{\infty}\right)^{\Gamma}$ and $S=B_{\infty} \in \mathfrak{M}\left(C^{*}\left(\widetilde{W}_{\infty}\right)^{\Gamma}\right)$, obtaining

2.26 Lemma. For every $f \in C_{0}(\mathbb{R})$ we get $f\left(D_{\infty}+B_{\infty}\right) \in C^{*}\left(\tilde{W}_{\infty}\right)^{\Gamma}$. If $f \in C([-\infty, \infty])$ then $f\left(D_{\infty}+\right.$ $\left.B_{\infty}\right) \in D^{*}\left(\widetilde{W}_{\infty}\right)^{\Gamma}$.

From Lemma 2.26 we learn that if $\chi$ is a chopping function then $\chi\left(D_{\infty}+B_{\infty}\right)$ is an element in $D^{*}\left(\widetilde{W}_{\infty}\right)^{\Gamma}$ and an involution modulo $C^{*}\left(\widetilde{W}_{\infty}\right)^{\Gamma}$. However, more is true.

2.27 Proposition. If $C_{\partial}$ and $C_{\infty}$ are as above, if $B_{\infty}$ is as in 2.24 and $\chi$ is a chopping function equal to the sign function on the spectrum of $D_{\partial}+C_{\partial}$, then $\chi\left(D_{\infty}+B_{\infty}\right)$ is an involution modulo $C^{*}\left(\widetilde{W} \subset \widetilde{W}_{\infty}\right)^{\Gamma}$.

Proof. Basically, we prove this by comparing $\chi\left(D_{\infty}+B_{\infty}\right)$ to the corresponding operator, $D_{\text {cyl }}+C_{\partial} \otimes \operatorname{Id}_{\mathbb{R}}$, on the the two-sided cylinder $\partial \tilde{W} \times \mathbb{R}$.

We start with some general comparison results. For simplification, we use the following notation:

- We use $D$ instead of $D_{\infty}$, and $\bar{D}$ for the corresponding (translation invariant) operator on $\mathbb{R} \times \partial \tilde{W}$.

- We use $C$ instead of $C_{\infty}$ and $\bar{C}$ for the translation invariant perturbation of $\bar{D}$ on $\mathbb{R} \times \partial W$; note that then $\bar{D}+\bar{C}$ is invertible.

- We use $B$ instead of $B_{\infty}$.

- We choose a cutoff function $\chi$ which is equal to \pm 1 on the spectrum of $\bar{D}+\bar{C}$, setting $e:=1-\chi^{2}$ then $e(\bar{D}+\bar{C})=0$.

- The subspace $[0, \infty) \times \partial \tilde{W}$ is contained isometrically in $\tilde{W}_{\infty}$ and in $\mathbb{R} \times \partial \tilde{W}$; we freely identify sections supported on this part of both manifolds. We employ the multiplication operator $P_{R}$ for $R \geq 0$; we remark that this is nothing but the orthogonal projection onto the sections supported on $[R, \infty) \times \partial \tilde{W}$. Once again, with a small abuse of notation, we employ the same symbols for this operator in $\widetilde{W}_{\infty}$. and in $\mathbb{R} \times \partial \widetilde{W}$. This way, in particular we consider an operator like $P_{0} f(\bar{D}) P_{R}$ as acting on $L^{2}\left(\tilde{W}_{\infty}\right)$ (instead of $\left.L^{2}(\partial \tilde{W} \times \mathbb{R})\right)$.

2.28 Lemma. For $f \in C_{0}(\mathbb{R}), P_{0} f(D) P_{0}-P_{0} f(\bar{D}) P_{0} \in C^{*}\left(\tilde{W} \subset \tilde{W}_{\infty}\right)^{\Gamma}$.

Proof. We know that $f(D) \in C^{*}\left(\tilde{W}_{\infty}\right)^{\Gamma}$ and $f(\bar{D}) \in C^{*}(\mathbb{R} \times \partial \tilde{W})^{\Gamma}$ and that $P_{0}$ is in $D^{*}$. So it only remains to check the support condition, i.e. to show that for each $\epsilon>0$ there is an $R>0$ such that $\left\|P_{0} f(D) P_{R}-P_{0} f(\bar{D}) P_{R}\right\|<\epsilon$ (then also its adjoint $P_{R} f(D) P_{0}-P_{R} f(\bar{D}) P_{0}$ has norm $\left.<\epsilon\right)$. For the latter, we approximate $f$ in supremum norm by a function $g$ whose Fourier transform has compact support (say in $[-R, R]$ ), and therefore approximate $f(D)$ in operator norm by $g(D)$ (and $f(\bar{D})$ by $g(\bar{D})$ ). The usual Fourier inversion formula then implies, using unit propagation speed for the wave operators of $D$ and $\bar{D}$, that $P_{0} g(D) P_{R}=P_{0} g(\bar{D}) P_{R}$, and the statement follows. 
We now generalize Lemma 2.28 to $D+B$. Note that we don't have unit propagation speed available. Instead, we give a proof which uses a comparison between $f(D)$ and $f(D+B)$.

2.29 Lemma. For $f \in C_{0}(\mathbb{R})$ and $B$ as in 2.24 we have that $P_{0} f(D+B) P_{0}-P_{0} f(\bar{D}+\bar{C}) P_{0} \in C^{*}(\tilde{W} \subset$ $\left.\tilde{W}_{\infty}\right)^{\Gamma}$.

Proof. For the following simple argument, using the Neumann series, we are grateful to the referee. We have to show that for $f \in C_{0}(\mathbb{R})$ it holds that $\lim _{R \rightarrow \infty}\left\|P_{R} f(D+B) P_{0}-P_{R} f(\bar{D}+\bar{C}) P_{0}\right\| \stackrel{R \rightarrow \infty}{\longrightarrow} 0$; the corresponding statement then also holds for the adjoint, involving $P_{0} f(D+B) P_{R}$.

Recall that if $X$ is a norm limit of bounded finite propagation operators then $\lim _{|R-S| \rightarrow \infty}\left\|\left(1-P_{S}\right) X P_{R}\right\|=$ 0 . Therefore, if $A, \bar{A}, B, \bar{B}$ are norm limits of $\Gamma$-equivariant finite propagation operators and if $P_{0} A P_{0}-$ $P_{0} \bar{A} P_{0}, P_{R} B P_{0}-P_{R} \bar{B} P_{0} \in C^{*}\left(\tilde{W} \subset \tilde{W}_{\infty}\right)^{\Gamma}$ in norm, then also

$$
\begin{aligned}
& P_{2 R} A B P_{0}-P_{2 R} \bar{A} \bar{B} P_{0}=\underbrace{\left(P_{2 R} A P_{R}-P_{2 R} \bar{A} P_{R}\right)}_{\stackrel{R \rightarrow \infty}{\longrightarrow}_{0}} P_{R} B P_{0}
\end{aligned}
$$

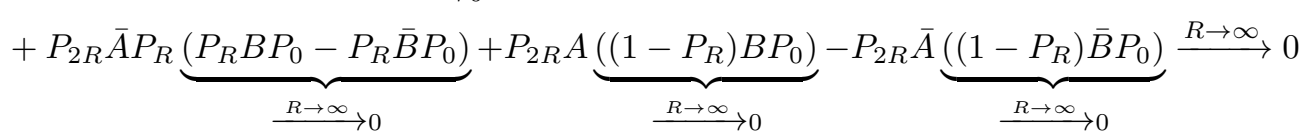

$$
\begin{aligned}
& \Longrightarrow P_{0} A B P_{0}-P_{0} \bar{A} \bar{B} P_{0} \in C^{*}\left(\tilde{W} \subset \tilde{W}_{\infty}\right)^{\Gamma} \text {. }
\end{aligned}
$$

Consequently, if the lemma is true for $f$ and $g$ then it is true for their product $f g$. As $C^{*}$-algebra, $C_{0}(\mathbb{R})$ is for any $\lambda>0$ generated by the two functions $x \mapsto(x+i \lambda)^{-1}$ and $x \mapsto(x-i \lambda)^{-1}$. This means that it suffices to treat the functions $x \mapsto(x+\lambda i)^{-1}$ and $x \mapsto(x-\lambda i)^{-1}$ for $\lambda>0$ large and we concentrate for notational convenience on the first.

As $\lambda$ is sufficiently large, we can then use the Neumann series

$$
(D+B+\lambda i)^{-1}=(D+\lambda i)^{-1} \sum_{k=0}^{\infty}\left(-B(D+\lambda i)^{-1}\right)^{k} ; \quad(\bar{D}+\bar{B}+\lambda i)^{-1}=(\bar{D}+\lambda i)^{-1} \sum_{k=0}^{\infty}\left(-\bar{B}(\bar{D}+\lambda i)^{-1}\right)^{k}
$$

By Lemma 2.28 $P_{0}(D+\lambda I)^{-1} P_{0}-P_{0}(\bar{D}+\lambda i)^{-1} P_{0} \in C^{*}\left(\tilde{W} \subset \tilde{W}_{\infty}\right)^{\Gamma}$ and by (2.24) $P_{0} B P_{0}-P_{0} \bar{B} P_{0} \in$ $C^{*}\left(\tilde{W} \subset \tilde{W}_{\infty}\right)^{\Gamma}$. We 2.30 we can pass to products and finite sums, showing that for each $N \in \mathbb{N}$ also

$$
P_{o}\left((D+\lambda i)^{-1} \sum_{k=0}^{N}\left(-B(D+\lambda i)^{-1}\right)^{k}\right) P_{0}-P_{o}\left((\bar{D}+\lambda i)^{-1} \sum_{k=0}^{N}\left(-\bar{B}(\bar{D}+\lambda i)^{-1}\right)^{k}\right) P_{0} \in C^{*}\left(\tilde{W} \subset \tilde{W}_{\infty}\right)^{\Gamma}
$$

Because of the norm convergence of the Neumann series then also

$$
P_{0}(D+B+\lambda i)^{-} 1 P_{0}-P_{0}(\bar{D}+\bar{B}+\lambda i)^{-1} P_{0} \in C^{*}\left(\tilde{W} \subset \tilde{W}_{\infty}\right)^{\Gamma}
$$

To finish the proof of Proposition 2.27 we only have to prove the support condition for $e(D+B)$, with $e(x)=1-\chi(x)^{2}$ as above, in particular $e \in C_{0}(\mathbb{R})$. It follows from Lemma 2.29 that

$$
P_{0} e(D+B) P_{0}-P_{0} e(\bar{D}+\bar{C}) P_{0} \in C^{*}\left(\tilde{W} \subset \tilde{W}_{\infty}\right)^{\Gamma},
$$

and by our choice of $\chi$ we have $e(\bar{D}+\bar{C})=0$. Finally, by finite propagation and the definition of $P_{0}$ also $P_{0} e(D+B)\left(1-P_{0}\right),\left(1-P_{0}\right) e(D+B)\left(1-P_{0}\right),\left(1-P_{0}\right) e(D+B) P_{0} \in C^{*}\left(\tilde{W} \subset \tilde{W}_{\infty}\right)^{\Gamma}$.

2.31 Definition. These considerations imply that there is a well defined coarse relative index class

$$
\operatorname{Ind}^{\mathrm{rel}}\left(D_{\infty}+C_{\infty}\right) \in K_{0}\left(C^{*}\left(\widetilde{W} \subset \widetilde{W}_{\infty}\right)^{\Gamma}\right) .
$$


It is obtained by the standard construction in coarse index theory: with an isometry $U: L^{2}\left(\widetilde{W}_{\infty}, \widetilde{E}^{-}\right) \rightarrow$ $L^{2}\left(\widetilde{W}_{\infty}, \widetilde{E}^{+}\right)$which is $\Gamma$-equivariant and which covers the identity in the $D^{*}$-sense (see [16, Section 1]) the operator $U \chi(D)_{+}$is invertible in $D^{*}\left(\widetilde{W}_{\infty}\right)^{\Gamma} / C^{*}\left(\widetilde{W} \subset \widetilde{W}_{\infty}\right)^{\Gamma}$ and therefore defines an element in $K_{1}$ of this $C^{*}$-algebra. Ind ${ }^{\text {rel }}\left(D_{\infty}+C_{\infty}\right)$ is its image under the boundary map of the associated long exact sequence in $K$-theory. We are also interested in the associated coarse index class

$$
\operatorname{Ind}(D, C):=c_{*}^{-1} \operatorname{Ind}^{\mathrm{rel}}\left(D_{\infty}+C_{\infty}\right) \in K_{0}\left(C^{*}(\widetilde{W})^{\Gamma}\right)
$$

Here we use the canonical inclusion $c: C^{*}(W)^{\Gamma} \rightarrow C^{*}\left(W \subset W_{\infty}\right)^{\Gamma}$ which induces an isomorphism in Ktheory, see [16, Lemma 1.9].

Note that the right hand side of 2.33 is just a notation; we have not really defined an operator $C$.

2.34 Proposition. If $B_{\infty}$ is the norm limit of $\Gamma$-equivariant bounded finite propagation operators, $C_{\partial}$ is a perturbation as above and we assume that $P_{0} B_{\infty} P_{0}-P_{0} C_{\infty} P_{0} \in C^{*}\left(\tilde{W}_{\infty} \subset \tilde{W}\right)^{\Gamma}$ then $\operatorname{Ind}^{\mathrm{rel}}\left(D_{\infty}+B_{\infty}\right)=$ $\operatorname{Ind}^{\mathrm{rel}}\left(D_{\infty}+C_{\infty}\right)$.

If $[0,1] \ni t \mapsto C_{\partial}^{t}$ is a continuous family of perturbations such that $D_{\partial}+C_{\partial}^{t}$ is invertible for all $t$, then $\operatorname{Ind}^{\mathrm{rel}}\left(D_{\infty}+C_{\infty}^{t}\right)$ is independent of $t$.

Finally, if $\widetilde{M} \rightarrow M$ is a $\Gamma$-Galois covering of a closed compact manifold $M$ and if $[0,1] \ni t \mapsto G_{t}$ is a continuous family of trivializing perturbations for $D$, as in Definition 2.10, then $\rho\left(D+G_{t}\right)$ and $\rho_{\Gamma}\left(D+G_{t}\right)$ are independent of $t$.

Proof. For the first two statements write either $A_{t}:=t B_{\infty}+(1-t) C_{\infty}$ or $A_{t}=C_{\infty}^{t}$. Then $[0,1] \ni t \mapsto D_{\infty}+A_{t}$ is norm resolvent continuous, as $\left(D_{\infty}+A_{t}+i\right)^{-1}-\left(D_{\infty}+A_{s}+i\right)^{-1}=\left(D_{\infty}+A_{t}+i\right)^{-1}\left(A_{s}-A_{t}\right)\left(D_{\infty}+A_{s}+i\right)^{-1}$ and $\left\|\left(D_{\infty}+A_{t}+i\right)^{-1}\right\| \leq 1$.

As the $C^{*}$-algebra $C[-\infty, \infty]$ of bounded continuous functions on $\mathbb{R}$ with limits at $\pm \infty$ is generated by $(x+i)^{-1},(x-i)^{-1}, 1$ and $\phi(x)=x / \sqrt{1+x^{2}}$ and the first two are taken care of by norm resolvent continuity, it remains to show that $t \mapsto \phi\left(D_{\infty}+A_{t}\right)$ is norm continuous to conclude that $t \mapsto \chi\left(D_{\infty}+A_{t}\right)$ is norm continuous for arbitrary $\chi \in C[-\infty, \infty]$.

To this end we write $\left\|\phi\left(D_{\infty}+A_{t}\right)-\phi\left(D_{\infty}+A_{s}\right)\right\|$ as $\left\|\phi(G)-\phi\left(G+S_{s, t}\right)\right\|$, with $G=D_{\infty}+A_{t}$ and $S_{s, t}=A_{s}-A_{t}$. We employ the following identity, see [2, Proposition 5.9] (this identity plays a fundamental role in the proof of Proposition 2.8.

$$
\begin{aligned}
\phi(G)-\phi\left(G+S_{s, t}\right)= & \frac{1}{\pi} \int_{1}^{+\infty} \frac{u}{\sqrt{u^{2}-1}} R(u) S_{s, t} R_{s, t}(u) d u \\
& +\frac{1}{\pi} \int_{1}^{+\infty} \frac{u}{\sqrt{u^{2}-1}} R(-u) S_{s, t} R_{s, t}(-u) d u
\end{aligned}
$$

where $R(u)=(G+i u)^{-1}$ and $R_{s, t}(u)=\left(G+S_{s, t}+i u\right)^{-1}$. Now, the norm of any of the two resolvent is bounded by $1 /|u|$; thus

$$
\left\|\phi(G)-\phi\left(G+S_{s, t}\right)\right\| \leq \frac{2}{\pi}\left\|A_{s}-A_{t}\right\|\left(\int_{1}^{\infty} \frac{u}{\sqrt{u^{2}-1}} \frac{1}{u^{2}} d u\right)
$$

This establishes the required continuity. In particular, for any chopping function $\chi, t \mapsto \chi\left(D_{\infty}+A_{t}\right)$ is a norm continuous family of involutions in $D^{*}\left(\tilde{W}_{\infty}\right)^{\Gamma} / C^{*}\left(\tilde{W}_{\infty} \subset \tilde{W}\right)^{\Gamma}$. By homotopy invariance of K-theory classes, the first two assertions follows.

A similar proof applies to the last statement once we observe that we can use the same chopping function taking values \pm 1 on the spectrum of all (invertible!) operators in the compact family $D_{\infty}+A_{t}, t \in[0,1]$. This follows from the norm continuity of $t \mapsto\left(D_{\infty}+A_{t}\right)^{-1}$, using

$$
\left(D_{\infty}+A_{s}\right)^{-1}=\left(D_{\infty}+A_{t}\right)^{-1}\left(1+\left(A_{s}-A_{t}\right)\left(D_{\infty}+A_{t}\right)^{-1}\right)^{-1}
$$

and a Neumann series argument for the last term. 


\subsubsection{Compatibility of index classes}

We now prove the compatibility of this relative index class in coarse geometry with the index class in the Mishchenko-Fomenko framework.

Let $D_{\infty}$ be a Dirac type operator on $\widetilde{W}_{\infty}$ and let $\mathcal{D}_{\infty}$ denote the associated operator on $W_{\infty}$ with coefficients in the Mishchenko bundle $\mathcal{F}_{\mathrm{M}}$. Let $\mathcal{C}_{\partial}$ be a trivializing perturbation for $\mathcal{D}_{\partial}$ and let $\mathcal{D}_{\infty}+\mathcal{C}_{\infty}$ the associated operator on $W_{\infty}$, obtained by applying the same construction explained above for the covering $\widetilde{W}$, see Definition 2.23. We concentrate directly on the case in which $\mathcal{C}_{\partial}$ is smoothing (as far as compatibility is concerned, this is the case we are interested in). By [8, Theorem 6.2] and [7, Theorem 10.1], $\left(\mathcal{D}_{\infty}+\mathcal{C}_{\infty}\right)^{+}$ is invertible modulo $\mathbb{K}\left(\mathcal{E}_{\mathrm{M}}\right)$. Further extensions of these results were subsequently given by Wahl in [24. Consequently, we get an index class

$$
\operatorname{Ind}_{\mathrm{MF}}^{b}\left(\mathcal{D}_{\infty}+\mathcal{C}_{\infty}\right) \in K_{n+1}\left(\mathbb{K}\left(\mathcal{E}_{\mathrm{M}}\right)\right)=K_{n+1}\left(C_{r}^{*} \Gamma\right)
$$

2.35 Remark. We can apply this construction to the manifold with boundary appearing in the definition of a cycle of $L_{n+1}(\mathbb{Z} \Gamma)$, choosing as a Dirac operator $\mathcal{D}$ the signature operator and as a trivializing perturbation for the boundary operator $\mathcal{D}_{\partial}$ the operator $\mathcal{C}_{f}$, the one induced by an homotopy equivalence $f$. We obtain an index class $\operatorname{Ind}_{\mathrm{MF}}^{b}\left(\mathcal{D}_{\infty}+\mathcal{C}_{f, \infty}\right) \in K_{n+1}\left(C_{r}^{*} \Gamma\right)$; this is precisely the index class we have considered in Section 2.1

We have already observed in [16] that there is an isomorphism $\mathbb{K}\left(\mathcal{E}_{\mathrm{M}}\right) \cong C^{*}\left(\widetilde{W} \subset \widetilde{W}_{\infty}\right)^{\Gamma}$. One can prove, as in [16, Section 2], the following proposition.

2.36 Proposition. Under the canonical isomorphism

$$
K_{n+1}\left(\mathbb{K}\left(\mathcal{E}_{\mathrm{M}}\right)\right) \cong K_{n+1}\left(C^{*}\left(\widetilde{W} \subset \widetilde{W}_{\infty}\right)^{\Gamma}\right)
$$

one has the equality

$$
\operatorname{Ind}_{\mathrm{MF}}^{b}\left(\mathcal{D}_{\infty}+\mathcal{C}_{\infty}\right)=\operatorname{Ind}^{\mathrm{rel}}\left(D_{\infty}+C_{\infty}\right)
$$

2.37 Remark. The index class $\operatorname{Ind}_{\mathrm{MF}}^{b}\left(\mathcal{D}_{\infty}+\mathcal{C}_{\infty}\right)$ heavily depends on the choice of trivializing perturbation $\mathcal{C}_{\partial}$. (Consequently, the same is true for $\operatorname{Ind}^{\text {rel }}\left(D_{\infty}+C_{\infty}\right)$.) Indeed, it is proved in [10, Theorem 6], inspired by 13 , that if $\mathcal{C}_{\partial}^{\prime}$ is a different perturbation then

$$
\operatorname{Ind}_{\mathrm{MF}}^{b}\left(\mathcal{D}_{\infty}+\mathcal{C}_{\infty}\right)-\operatorname{Ind}_{\mathrm{MF}}^{b}\left(\mathcal{D}_{\infty}+\mathcal{C}_{\infty}^{\prime}\right)=\left[\mathcal{P}^{\prime}-\mathcal{P}\right] \quad \text { in } \quad K_{\operatorname{dim} W}\left(C_{r}^{*} \Gamma\right)
$$

with $\left[\mathcal{P}^{\prime}-\mathcal{P}\right]$ the difference class of the two projections $\mathcal{P}=\chi_{[0, \infty)}\left(\mathcal{D}_{\partial}+\mathcal{C}\right)$ and $\mathcal{P}^{\prime}=\chi_{[0, \infty)}\left(\mathcal{D}_{\partial}+\mathcal{C}^{\prime}\right)$.

\section{Delocalized APS-index theorem for perturbed operators}

Let $\left(\widetilde{W}, g_{\widetilde{W}}\right)$ be an even dimensional Riemannian orientable manifold with boundary $\partial \widetilde{W}$. Assume that $\Gamma$ acts freely, cocompactly and isometrically on $\widetilde{W}$. We consider the associated manifold with cylindrical ends $\widetilde{W}_{\infty}$. Let $C_{\partial}$ be a trivializing perturbation as before (i.e. the norm limit of $\Gamma$-equivariant bounded self-adjoint finite propagation operators such that $D_{\partial}+C_{\partial}$ is $L^{2}$-invertible) and let $C_{\infty}$ be the associated perturbation on $\widetilde{W}_{\infty}$, as in Section 2.3.1. Recall the coarse index class $\operatorname{Ind}(D, C) \in K_{0}\left(C^{*}(\widetilde{W})^{\Gamma}\right)$ defined in $(2.33)$. Our main tool in this paper will be the following "delocalized APS-index theorem for perturbed operators".

3.1 Theorem. The following equality holds

$$
\iota_{*}(\operatorname{Ind}(D, C))=j_{*}\left(\rho\left(D_{\partial}+C_{\partial}\right)\right) \quad \text { in } \quad K_{0}\left(D^{*}(\widetilde{W})^{\Gamma}\right) .
$$

Here, we use $j: D^{*}(\widetilde{\widetilde{W}})^{\Gamma} \rightarrow D^{*}(\widetilde{W})^{\Gamma}$ induced by the inclusion $\partial \widetilde{W} \rightarrow \widetilde{W}$ and $\iota: C^{*}(\widetilde{W})^{\Gamma} \rightarrow D^{*}(\widetilde{W})^{\Gamma}$ the inclusion. 
3.3 Corollary. By functoriality, using the canonical $\Gamma-m a p u: \widetilde{W} \rightarrow E \Gamma$ we have

$$
\iota_{*} u_{*}(\operatorname{Ind}(D, C))=\rho_{\Gamma}\left(D_{\partial}+C_{\partial}\right) \quad \text { in } \quad K_{0}\left(D_{\Gamma}^{*}\right) .
$$

If we define $\operatorname{Ind}_{\Gamma}(D, C):=u_{*}(\operatorname{Ind}(D, C))$ in $K_{0}\left(C_{\Gamma}^{*}\right)$ then the last equation reads

$$
\iota_{*}\left(\operatorname{Ind}_{\Gamma}(D, C)\right)=\rho_{\Gamma}\left(D_{\partial}+C_{\partial}\right) \quad \text { in } \quad K_{0}\left(D_{\Gamma}^{*}\right) .
$$

We prove these results in Section 5 .

3.5 Remark. Consider $B_{\infty}$, a bounded self-adjoint $\Gamma$-equivariant odd operator on $W_{\infty}$ satisfying (2.24), i.e. $B_{\infty}$ is a norm limit of $\Gamma$-equivariant finite propagation operators and $P_{0} B_{\infty} P_{0}-P_{0} C_{\infty} P_{0} \in C^{*}\left(\tilde{W}^{C} \subset W_{\infty}\right)^{\Gamma}$. By Proposition 2.27, we have the coarse index class $\operatorname{Ind}(D, B):=c_{*}^{-1} \operatorname{Ind}^{\mathrm{rel}}\left(D_{\infty}+B_{\infty}\right) \in K_{n+1}\left(C^{*}(\widetilde{W})^{\Gamma}\right)$. Since by Proposition $2.34 \operatorname{Ind}(D, B)=\operatorname{Ind}(D, C)$, we also have

$$
\iota_{*}\left(\operatorname{Ind}_{\Gamma}(D, B)\right)=\rho_{\Gamma}\left(D_{\partial}+C_{\partial}\right) .
$$

The above remark brings us immediately to a version of bordism invariance for $\rho$-classes:

3.7 Corollary. Let $\widetilde{M}_{1}$ and $\widetilde{M}_{2}$ be two free cocompact $\Gamma$-manifolds of dimension $n$. Let $D_{j}$ be a $\Gamma$-equivariant Dirac type operator on $\widetilde{M}_{j}, C_{j} \in C^{*}\left(\widetilde{M}_{j}\right)^{\Gamma}$ a trivializing perturbations $(j=1,2)$. Assume that $D_{1}$ and $D_{2}$ are bordant, i.e. there exists a manifold $\widetilde{W}$ with free cocompact $\Gamma$-action such that $\partial \widetilde{W}=\widetilde{M}_{1} \sqcup \widetilde{M}_{2}$ and $D$ a $\Gamma$-equivariant Dirac type operator on $W$ with product structure near the boundary (in the sense of Dirac type operators) and with boundary operator $D_{1} \sqcup-D_{2}$. Moreover, let $B_{\infty}$ be a global perturbation which is a norm limit of finite propagation operators and such that $P_{0} B_{\infty} P_{0}-P_{0} C_{\infty}^{\sqcup} P_{0} \in C^{*}\left(\tilde{W} \subset \tilde{W}_{\infty}\right)^{\Gamma}$, where $C_{\infty}^{\sqcup}:=C_{1, \infty} \sqcup\left(-C_{2, \infty}\right)$.

Assume in addition that $\operatorname{Ind}_{\Gamma}(D, B)=0$ in $K_{n+1}\left(C_{\Gamma}^{*}\right)$. Note that this is satisfied e.g. if $B_{\infty}$ is a trivializing perturbation of $D$, i.e. if $D+B_{\infty}$ is invertible. Then

$$
\rho_{\Gamma}\left(D_{1}+C_{1}\right)=\rho_{\Gamma}\left(D_{2}+C_{2}\right) \in K_{n+1}\left(D_{\Gamma}^{*}\right)
$$

Proof. The rho-class is additive for disjoint union. By assumption, $\operatorname{Ind}_{\Gamma}(D, B)=0$. The assertion now follows directly from 3.6 .

3.8 Remark. We can now go back to Example 2.20 and explain why

$$
\rho_{\Gamma}\left(D+\mathcal{S}_{0}\right)=\rho_{\Gamma}\left(D+\mathcal{S}_{1}\right) \text { if } \mathcal{S}_{0}, \mathcal{S}_{1} \text { are two symmetric trivializing perturbations. }
$$

Recall that we are considering $\widetilde{M} \rightarrow M$, a $\Gamma$-cover such that the differential-form Laplacian on the covering is $L^{2}$-invertible in degree $m$. The above equality takes place in $K_{\operatorname{dim} M+1}\left(D_{\Gamma}^{*}\right)$.

We consider $[0,1] \times \widetilde{M}$ with associated manifold with cylindrical ends. By the delocalized APS-index theorem 3.1 we have that

$$
\rho_{\Gamma}\left(D+\mathcal{S}_{0}\right)-\rho_{\Gamma}\left(D+\mathcal{S}_{1}\right)=\iota_{*}\left(\operatorname{Ind}_{\Gamma}\left(D_{\infty}+\mathcal{S}_{\infty}^{0,1}\right)\right)
$$

with $\mathcal{S}_{\infty}^{0,1}$ the grafted perturbation associated to $\mathcal{S}_{0}$ on $\{0\} \times \widetilde{M}$ and $\mathcal{S}_{1}$ on $\{1\} \times \widetilde{M}$. Using in a fundamental way the hypothesis that $\mathcal{S}^{j}$ are symmetric we have, see [9, Proposition 4.4], 23]

$$
\operatorname{Ind}_{\Gamma}\left(D_{\infty}+\mathcal{S}_{\infty}^{0,1}\right)=\operatorname{Ind}_{\Gamma}\left(D_{\infty}+\mathcal{S}_{\infty}^{0,0}\right) \text { in } K_{*}\left(C_{r}^{*} \Gamma\right)=K_{*}\left(C_{\Gamma}^{*}\right)
$$

Thus

$$
\begin{aligned}
\rho_{\Gamma}\left(D+\mathcal{S}_{0}\right)-\rho_{\Gamma}\left(D+\mathcal{S}_{1}\right) & =\left(\rho_{\Gamma}\left(D+\mathcal{S}_{0}\right)-\rho_{\Gamma}\left(D+\mathcal{S}_{1}\right)\right)-\left(\rho_{\Gamma}\left(D+\mathcal{S}_{0}\right)-\rho_{\Gamma}\left(D+\mathcal{S}_{0}\right)\right) \\
& =\iota_{*}\left(\operatorname{Ind}_{\Gamma}\left(D_{\infty}+\mathcal{S}_{\infty}^{0,1}\right)\right)-\iota_{*}\left(\operatorname{Ind}_{\Gamma}\left(D_{\infty}+\mathcal{S}_{\infty}^{0,0}\right)\right)=0 .
\end{aligned}
$$


3.9 Remark. As in [16, it is plausible that the methods which prove the delocalized APS-index theorem for perturbed operators, also yield a secondary partitioned manifold index theorem 11 We leave the precise formulation and proof to future investigations. A potential application of such a theorem would be the following: assume that $M_{1}, M_{2}$ are two oriented complete Riemannian manifolds with free proper isometric $\Gamma$-action and with $\Gamma$-invariant and $\Gamma$-cocompact separating hypersurfaces $N_{1} \subset M_{1}, N_{2} \subset M_{2}$ with product neighborhoods. Let $f: M_{1} \rightarrow M_{2}$ be a $\Gamma$-equivariant homotopy equivalence which restricts to a homotopy equivalence $f \mid: N_{1} \rightarrow N_{2}$. Assume that $f$ coarsely preserves the signed distance to the hypersurfaces, i.e. that the two functions $d_{1}, d_{2} \circ f: M_{1} \rightarrow \mathbb{R}$ with $d_{1}(x)=\epsilon_{1}(x) d\left(x, N_{1}\right)$ and $d_{2}(y)=\epsilon_{2}(y) d\left(y, N_{2}\right)$ are coarsely equivalent to each other. Here $\epsilon_{1}(x)= \pm 1$ depending whether $x$ lies in the positive or negative half of $M$.

We call such a situation a "partitioned manifold homotopy equivalence".

In this situation, possibly with further hypothesis on $f$, one should obtain a partitioned manifold $\rho$-class $\rho^{\mathrm{pm}}(f) \in K_{*}\left(D_{\Gamma}^{*}\right)$. Similarly, we defined in $2.18 \rho_{\Gamma}\left(\left.f\right|_{N_{1}}\right) \in K_{*}\left(D_{\Gamma}^{*}\right)$. The secondary partitioned manifold index theorem would claim that these two classes coincide.

As a potential application, assume that $f: N_{1} \rightarrow N_{2}$ and $g: X_{1} \rightarrow N_{2}$ are homotopy equivalences and that $\rho_{\Gamma}(f) \neq \rho_{\Gamma}(g)$ so that $\left(N_{1} \stackrel{f}{\rightarrow} N_{2}\right)$ is not h-cobordant to $\left(X_{1} \stackrel{g}{\rightarrow} N_{2}\right)$. Then, also between the stabilizations $\left(N_{1} \times \mathbb{R} \stackrel{f \times \mathrm{id}_{\mathbb{R}}}{\longrightarrow} N_{2} \times \mathbb{R}\right)$ and $\left(X_{1} \times \mathbb{R} \stackrel{g \times \mathrm{id}_{\mathbb{R}}}{\longrightarrow} N_{2} \times \mathbb{R}\right)$ there is no Riemannian h-cobordism such that the inclusion maps of the boundaries are continuous coarse homotopy equivalences. Otherwise, we expect $\rho^{\mathrm{pm}}\left(f \times \operatorname{id}_{\mathbb{R}}\right)=\rho^{\mathrm{pm}}\left(g \times \operatorname{id}_{\mathbb{R}}\right)$; however, by the secondary index theorem, this would contradict the hypothesis $\rho_{\Gamma}(f) \neq \rho_{\Gamma}(g)$.

\section{Mapping the surgery sequence to K-Theory}

\subsection{The structure set and the map $\rho_{\Gamma}$}

Let $V$ be an $n$-dimensional smooth closed oriented manifold with fundamental group $\Gamma, n>4$.

4.1 Definition. The structure set $\mathcal{S}^{h}(V)$ consists of equivalence classes $[M \stackrel{f}{\rightarrow} V]$, where $M$ is a smooth closed oriented manifold, and $f$ is an homotopy equivalence. $\left(M_{1} \stackrel{f_{1}}{\longrightarrow} V\right)$ and $\left(M_{2} \stackrel{f_{2}}{\longrightarrow} V\right)$ are equivalent if there is an h-cobordism $X$ between $M_{1}$ and $M_{2}$ and a map $F: X \rightarrow V \times[0,1]$ (necessarily a homotopy equivalence) such that $\left.F\right|_{M_{1}}=f_{1}$ and $\left.F\right|_{M_{2}}=f_{2}$.

Let $V$ be as above. Let $u: V \rightarrow B \Gamma$ be the classifying map for the universal cover of $V$ and let $\tilde{u}: \widetilde{V} \rightarrow E \Gamma$ be a $\Gamma$-equivariant lift of $u$. Let $M \stackrel{f}{\rightarrow} V$ be a cycle representing an element $[M \stackrel{f}{\rightarrow} V]$ in $\mathcal{S}^{h}(V)$. As in example 2.18 we consider $Z=M \sqcup(-V)$ with the obvious classifying map $u_{Z}: Z \rightarrow B \Gamma$ induced by $u$ and by $u \circ f$. We observe that $Z$ comes with a map $\phi$ to $V, \phi:=f \sqcup\left(-\operatorname{Id}_{V}\right)$, which is covered by a $\Gamma$-equivariant $\operatorname{map} \tilde{\phi}: \widetilde{Z} \rightarrow \widetilde{V}$.

4.2 Definition. We define a map $\rho: \mathcal{S}^{h}(V) \mapsto K_{n+1}\left(D^{*}(\widetilde{V})^{\Gamma}\right)$ as follows

$$
\mathcal{S}^{h}(V) \ni[M \stackrel{f}{\rightarrow} V] \rightarrow \rho(f) \in K_{n+1}\left(D^{*}(\widetilde{V})^{\Gamma}\right)
$$

where we recall that $D$ is the signature operator on the covering $\widetilde{Z}:=u_{Z}^{*} E \Gamma, C_{f}$ is the smoothing trivializing perturbation constructed using the homotopy equivalence $f$ and $\rho(f):=\tilde{\phi}_{*}\left(\rho\left(D+C_{f}\right)\right) \in K_{n+1}\left(D^{*}(\widetilde{V})^{\Gamma}\right)$.

4.4 Definition. We define

$$
\rho_{\Gamma}: \mathcal{S}^{h}(V) \rightarrow K_{n+1}\left(D_{\Gamma}^{*}\right) ;[M \stackrel{f}{\rightarrow} V] \mapsto \rho_{\Gamma}(f)
$$

\footnotetext{
${ }^{1}$ We take this opportunity to point out that the secondary partitioned manifold index theorem proved in 16 can in fact be sharpened, with essentially the same proof, from an equality of universal rho-classes to an equality of rho-classes. We thank Vito Felice Zenobi and Rudolf Zeidler for alerting us of this sharpening.
} 
where we recall that

$$
\rho_{\Gamma}(f):=\rho_{\Gamma}\left(D+C_{f}\right):=\left(\tilde{u}_{Z}\right)_{*}\left(\rho\left(D+C_{f}\right)\right)=\tilde{u}_{*}(\rho(f)) .
$$

If necessary, we shall denote the right hand sides of $\left[4.3\right.$ and $\left[4.5\right.$ as $\rho[M \stackrel{f}{\rightarrow} V]$ and $\rho_{\Gamma}[M \stackrel{f}{\rightarrow} V]$ respectively.

From now on we assume our manifold $V$ to be odd dimensional.

4.6 Remark. Notice that we follow here different conventions with respect to [4]; indeed we follow the conventions of [15], slightly modified as in [25]. In particular, the operator we use on an odd dimensional manifold is not the odd signature operator as in Atiyah, Patodi, Singer (and the one used by topologists to define the signature K-homology class, compare [19]). It is rather the direct sum of two (unitarily equivalent) versions of this operator, and comes up as the boundary operator of the signature operator on an even dimensional manifold. This results in a possible loss of two-torsion information. On the other hand, with this modification our diagrams commute without inverting 2 .

However, a factor of 2 would with our conventions show up in the same portion of the sequence for an even dimensional manifold $V$.

It would be intersting to improve the constructions of our invariants, in particular the rho-invariant, so that they deal with the signature operator on odd dimensional manifolds as of Atiyah-Patodi-Singer (potentially containing additional 2-torsion information). This involves understanding how the HilsumSkandalis-Wahl perturbation can be made compatible with the splitting of the operator alluded to above. It also involves establishing bordism invariance of the rho-invariant to be obtained that way. This is a nontrivial task, as shows [19, where the corresponding problem of bordism invariance of the K-homology class is solved.

Finally, we believe that our diagram can be extended to the left, provided that one inverts 2 (and multiplies all maps with the appropriate powers of 2, similar to [4]), and in such a situation the powers of 2 discussed above will inevitably appear to make all squares commutative.

4.7 Proposition. The maps

$$
\mathcal{S}^{h}(V) \ni[M \stackrel{f}{\rightarrow} V] \mapsto \rho_{\Gamma}(f) \in K_{n+1}\left(D_{\Gamma}^{*}\right) \quad \text { and } \quad \mathcal{S}^{h}(V) \ni[M \stackrel{f}{\rightarrow} V] \mapsto \rho(f) \in K_{n+1}\left(D^{*}(\widetilde{V})^{\Gamma}\right)
$$

are well defined.

Proof. This follows from the work [25] of Charlotte Wahl and our delocalized APS index theorem. Let us first prove that $\rho(f) \in K_{0}\left(D^{*}(\widetilde{V})^{\Gamma}\right)$ is well defined. We assume as before that $\left[M_{1} \stackrel{f_{1}}{\longrightarrow} V\right]=\left[M_{2} \stackrel{f_{2}}{\longrightarrow} V\right]$ in $\mathcal{S}^{h}(V)$ and let $F: X \rightarrow V \times[0,1]$ be the h-cobordism realizing this equality; we set again $W=X \sqcup V \times[0,1]$. Then there is a map $\Phi: W \rightarrow V$ given by $F \sqcup \operatorname{id}_{V \times[0,1]}: W \rightarrow V \times[0,1]$, followed by the projection $\pi_{1}$ onto the the first factor of $V \times[0,1]$; i.e. $\Phi=\pi_{1} \circ\left(F \sqcup \mathrm{id}_{V \times[0,1]}\right)$. Let $\phi_{1}=f_{1} \sqcup\left(-\operatorname{Id}_{V}\right): M_{1} \sqcup(-V) \rightarrow V$ and similarly for $\phi_{2}$. Set as before $Z_{1}:=M_{1} \cup(-V)$ and $Z_{2}:=M_{2} \cup(-V)$. We have observed that these manifolds come with classifying maps $u \circ \phi_{j}$ into $B \Gamma$ and we have denote by $\widetilde{Z}_{j}$ the corresponding $\Gamma$-covers. As before, we consider the $\Gamma$-equivariant lifts $\tilde{\phi}_{j}: \widetilde{Z}_{j} \rightarrow \widetilde{V}$. Recall now that $\rho\left(f_{1}\right) \in K_{0}\left(D^{*}(\widetilde{V})^{\Gamma}\right)$ is obtained by pushing forward through $\left(\tilde{\phi}_{1}\right)_{*}$ the rho-class of $\widetilde{Z}_{1}$, denoted $\rho\left(D_{\widetilde{Z}_{1}}+C_{f_{1}}\right) \in K_{0}\left(D^{*}\left(\widetilde{Z}_{1}\right)^{\Gamma}\right)$. Similarly $\rho\left(f_{2}\right)=\left(\tilde{\phi}_{2}\right)_{*} \rho\left(D_{\widetilde{Z}_{2}}+C_{f_{2}}\right)$. Clearly $\Phi$ restricted to $M_{1} \sqcup(-V)=: Z_{1}$ is $\phi_{1}$ and similarly for $\Phi$ restricted to $M_{1} \sqcup(-V)=: Z_{2}$. We can now apply $\Phi_{*}$ to the delocalized APS index formula for $W$, which we write with self-explanatory notation as

$$
\left(\iota_{\widetilde{W}}\right)_{*} \operatorname{Ind}\left(D_{\widetilde{W}}, C_{1,2}\right)=\left(j_{1}\right)_{*} \rho\left(D_{\widetilde{Z}_{1}}+C_{f_{1}}\right)-\left(j_{2}\right)_{*} \rho\left(D_{\widetilde{Z}_{2}}+C_{f_{2}}\right)
$$

with $j_{1}$ and $j_{2}$ the obvious inclusions. This equality takes place in $K_{0}\left(D^{*}(\widetilde{W})^{\Gamma}\right)$. Now, Wahl proves in $[25$, Theorem 8.4], extending the work of Hilsum-Skandalis to manifolds with cylindrical ends, that the existence of the map $F$ entering into the h-cobordism, i.e. of a global homotopy equivalence, implies the vanishing of the index class obtained by grafting the two smoothing perturbations $C_{f_{j}}$ on the cylindrical ends (Wahl works 
in the Mishchenko-Fomenko framework but since we know that this is equivalent to the coarse framework we can directly state her results in the way we just have). Since the latter is precisely the index class that was denoted $\operatorname{Ind}\left(D_{\widetilde{W}}, C_{1,2}\right)$ in the left hand side of 4.8 , we obtain at once that

$$
0=\left(j_{1}\right)_{*} \rho\left(D_{\widetilde{Z}_{1}}+C_{f_{1}}\right)-\left(j_{2}\right)_{*} \rho\left(D_{\widetilde{Z}_{2}}+C_{f_{2}}\right) \text { in } K_{0}\left(D^{*}(\widetilde{W})^{\Gamma}\right) .
$$

We now apply $\Phi_{*}$ to both sides and we obtain the following equality in $K_{0}\left(D^{*}(\tilde{V})^{\Gamma}\right)$ :

$$
0=\Phi_{*}\left(\left(j_{1}\right)_{*} \rho\left(Z_{1}\right)\right)-\Phi_{*}\left(\left(j_{2}\right)_{*} \rho\left(Z_{2}\right)\right)
$$

However, by functoriality, the right hand side is exactly $\rho\left(f_{1}\right)-\rho\left(f_{2}\right)$. Thus $0=\rho\left(f_{1}\right)-\rho\left(f_{2}\right)$ and we are done.

This argument also proves that $\rho_{\Gamma}$ is well defined; indeed it suffices to apply $\tilde{u}_{*}: K_{0}\left(D^{*}(\widetilde{V})^{\Gamma}\right) \rightarrow K_{0}\left(D_{\Gamma}^{*}\right)$ to the equality $0=\rho\left(f_{1}\right)-\rho\left(f_{2}\right)$ and recall that $\tilde{u}_{*}\left(\rho\left(f_{j}\right)\right)=\rho_{\Gamma}\left(f_{j}\right)$. See the remark after $(2.19)$.

\subsection{The set $\mathcal{N}(V)$ and the map $\beta_{\Gamma}: \mathcal{N}(V) \rightarrow K_{n}(B \Gamma)$}

Let $V$ be an $n$-dimensional smooth closed oriented manifold with fundamental group $\Gamma$. We assume that $n>4$ and that it is odd. Let $u: V \rightarrow B \Gamma$ be the classifying map for the universal covering of $V$ and let $\widetilde{V}:=u^{*} E \Gamma$.

4.9 Definition. $\mathcal{N}(V)$ is the set of normal maps. Its elements are pairs $[M \stackrel{f}{\rightarrow} V]$ where $M$ is again a closed smooth oriented manifold and $f$ is a degree 1 normal map. Two such pairs are equivalent if there is a normal cobordism between them (for all this compare for example [4, Section 5] and the references therein).

The map $\beta_{\Gamma}$ is the composition of $\beta: \mathcal{N}(V) \rightarrow K_{*}(V)$ and the group homomorphism $u_{*}: K_{*}(V) \rightarrow$ $K_{*}(B \Gamma)$ induced by the classifying map of $V$. Here $\beta$ maps $[M \stackrel{f}{\rightarrow} V]$ in $\mathcal{N}(V)$ to $f_{*}\left[D_{M}\right]-\left[D_{V}\right] \in K_{*}(V)$, where $\left[D_{M}\right],\left[D_{V}\right]$ are the K-homology classes of the signature operators. The map $\beta: \mathcal{N}(V) \rightarrow K_{*}(V)$ already appears in the work of Higson and Roe where it is proved to be well defined.

\subsection{Mapping the surgery sequence to the Higson-Roe sequence}

4.10 Theorem. Let $V$ be an $n$-dimensional smooth closed oriented manifold with fundamental group $\Gamma$. We assume that $n>4$ is odd. Then there is a commutative diagram with exact rows

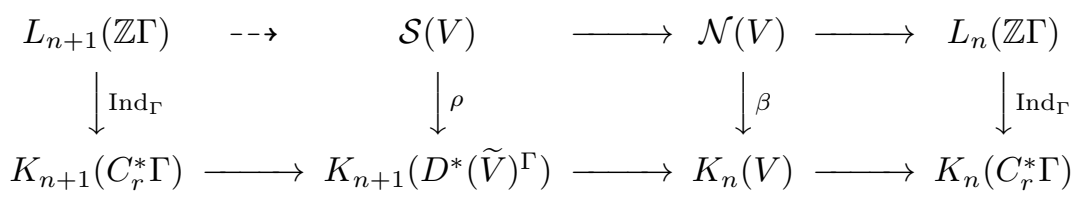

By employing the classifying map $u: V \rightarrow B \Gamma$ for the universal cover $\tilde{V}$ of $V$ we also get a commutative diagram mapping into the universal Higson-Roe surgery sequence:

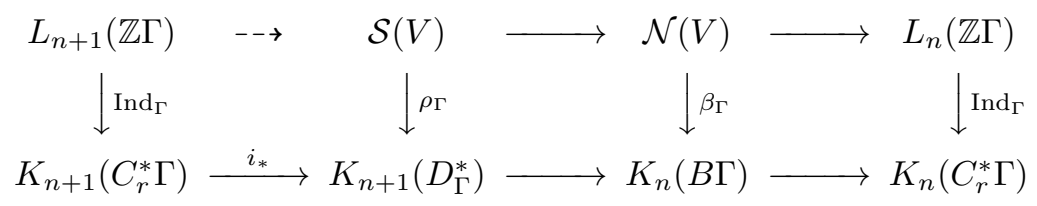

Proof. Thanks to the work of Wahl (see Theorem 2.1) and Proposition 2.34 we know that the index homomorphism Ind: $L_{*}(\mathbb{Z} \Gamma) \rightarrow K_{*}\left(C_{r}^{*} \Gamma\right)$ is well defined ${ }^{2}$. By Proposition $4.7, \rho$ is well defined and we know from 4. Definition 5.2] that $\beta$ is also well defined. We now proceed to prove the commutativity of the squares.

\footnotetext{
${ }^{2}$ Wahl concentrates on the case $*$ even, but for this particular result her arguments apply, with a minimum amount of work, to the case $*$ odd.
} 
Recall first of all the meaning of exactness of the surgery sequence at $\mathcal{S}(V)$, see for example [4]. Let $[M \stackrel{f}{\rightarrow} V] \in \mathcal{S}(V)$ and let $a \in L_{n+1}(\mathbb{Z} \Gamma)$. Then $a$ can be represented by $\left(W, F, M \times[0,1], u_{M}: M \rightarrow B \Gamma\right)$ with $F: W \rightarrow M \times[0,1]$ a normal map of degree one between manifolds with boundary and $u_{M}=u \circ f$. Here $W$ is a manifold with boundary with two boundary components $\partial_{0} W$ and $\partial_{1} W, \partial_{0} F:=\left.F\right|_{\partial_{0} W}: \partial_{0} W \rightarrow$ $\{0\} \times M$ is a diffeomorphism and $\partial_{1} F:=\left.F\right|_{\partial_{1} W}: \partial_{1} W \rightarrow\{1\} \times M$ is a homotopy equivalence. For later use we set $X:=W \sqcup M \times[0,1]$ and we remark that $X$ comes with a natural map $u_{f}: X \rightarrow V$ with $u_{f}:=f \circ\left(\pi_{1}\left(F \circ \mathrm{id}_{M \times[0,1]}\right)\right)$ and $\pi_{1}$ the projection onto the first factor. The action of $L_{n+1}(\mathbb{Z} \Gamma)$ on $\mathcal{S}(V)$ is as follows: if $a \in L_{n+1}(\mathbb{Z} \Gamma)$ and $[M \stackrel{f}{\rightarrow} V] \in \mathcal{S}(V)$ then

$$
a \cdot[M \stackrel{f}{\rightarrow} V]:=\left[\partial_{1} W \stackrel{f \circ \partial_{1} F}{\longrightarrow} V\right] .
$$

Exactness of the surgery sequence at $\mathcal{S}(V)$ means the following: two elements in the structure set belong to the same orbit under the action of the group $L_{n+1}(\mathbb{Z} \Gamma)$ if and only if their images in $\mathcal{N}(V)$ coincide. By definition, in this situation where we deal with group actions instead of homomorphisms, commutativity of the first square means

$$
\rho(a \cdot[M \stackrel{f}{\rightarrow} V])-\rho[M \stackrel{f}{\rightarrow} V]=i_{*} \operatorname{Ind}(a) \text { in } \quad K_{n+1}\left(D^{*}(\widetilde{V})^{\Gamma}\right)
$$

i.e. that

$$
\rho\left[\partial_{1} W \stackrel{f \circ \partial_{1} F}{\rightarrow} V\right]-\rho[M \stackrel{f}{\rightarrow} V]=i_{*} \operatorname{Ind}(a) .
$$

The homomorphism $i_{*}$ appearing on the right hand side is, up to a canonical isomorphism, the map induced by the inclusion $C^{*}(\widetilde{V})^{\Gamma} \rightarrow D^{*}(\widetilde{V})^{\Gamma}$ whereas the index class $\operatorname{Ind}(a)$ can be taken to be the push-forward, from $K_{n+1}\left(C^{*}(\widetilde{X})^{\Gamma}\right)$ to $K_{n+1}\left(C^{*}(\widetilde{V})^{\Gamma}\right)$, of the index class defined by the perturbed signature operator on $\widetilde{X}$ :

$$
\operatorname{Ind}(a)=\left(\tilde{u}_{f}\right)_{*}\left(\operatorname{Ind}\left(D_{\widetilde{X}}+C_{\partial_{0} F, \partial_{1} F}\right)\right)
$$

with $\tilde{u}_{f}$ the $\Gamma$-map covering $u_{f}: X \rightarrow V$. Now, the proof of [25, Proposition 7.1], together with Proposition 2.34 shows that we have the following identity in $K_{*+1}\left(D^{*}(\widetilde{V})^{\Gamma}\right)$ :

$$
\rho[L \stackrel{f \circ g}{\longrightarrow} V]+\tilde{f}_{*}\left(\rho\left[M \stackrel{\mathrm{Id}_{M}}{\longrightarrow} M\right]\right)=\tilde{f}_{*}(\rho[L \stackrel{g}{\rightarrow} M])+\rho[M \stackrel{f}{\rightarrow} V] .
$$

In order to prove Equation (4.14) we consider the manifold $Y:=L \sqcup(-M) \sqcup M \sqcup(-V)$ and the natural inclusions

$Z_{1}:=L \sqcup(-M) \stackrel{j_{1}}{\rightarrow} Y, \quad Z_{2}:=M \sqcup(-V) \stackrel{j_{2}}{\rightarrow} Y, \quad T_{1}:=L \sqcup(-M) \stackrel{i_{1}}{\rightarrow} Y \quad$, and $\quad T_{2}:=M \sqcup(-V) \stackrel{i_{2}}{\longrightarrow} Y$.

One gets the invertible operators $D_{\widetilde{Z}_{1}}+C_{g}$ and $D_{\widetilde{Z}_{2}}+C_{f}$ and the associated rho classes

$$
\rho\left(D_{\widetilde{Z}_{1}}+C_{g}\right) \in K_{n+1}\left(D^{*}\left(\widetilde{Z}_{1}\right)^{\Gamma}\right) ; \quad \rho\left(D_{\widetilde{Z}_{2}}+C_{f}\right) \in K_{n+1}\left(D^{*}\left(\widetilde{Z}_{2}\right)^{\Gamma}\right) .
$$

Similarly, we obtain the invertible operators $D_{\widetilde{T}_{1}}+C_{f \circ g}$ and $D_{\widetilde{T}_{2}}+C_{\text {Id }}$ and the rho classes

$$
\rho\left(D_{\widetilde{T}_{1}}+C_{f \circ g}\right) \in K_{n+1}\left(D^{*}\left(\widetilde{T}_{1}\right)^{\Gamma}\right) ; \quad \rho\left(D_{\widetilde{T}_{2}}+C_{\mathrm{id}}\right) \in K_{n+1}\left(D^{*}\left(\widetilde{T}_{2}\right)^{\Gamma}\right)
$$

We claim that

$$
\left(\tilde{j}_{1}\right)_{*}\left(\rho\left(D_{\widetilde{Z}_{1}}+C_{g}\right)\right)+\left(\tilde{j}_{2}\right)_{*}\left(\rho\left(D_{\widetilde{Z}_{2}}+C_{f}\right)\right)=\left(\tilde{i}_{1}\right)_{*}\left(\rho\left(D_{\widetilde{T}_{1}}+C_{f \circ g}\right)\right)+\left(\tilde{i}_{2}\right)_{*}\left(\rho\left(D_{\widetilde{T}_{2}}+C_{\mathrm{Id}}\right)\right)
$$

in the group $K_{n+1}\left(D^{*}(\tilde{Y})^{\Gamma}\right)$. Indeed, by a simple functoriality argument, both sides are defined by rho classes of suitable perturbations of the signature operator on $\tilde{Y}$; Wahl shows in the proof of [25, Proposition 7.1] that there is a continuous family of trivializing perturbations, [0,1] $\ni t \rightarrow G_{t}$, interpolating from the perturbation 
defining the left hand side to the perturbation defining the right hand side. Thanks to Proposition 2.34 we then obtain immediately (4.15). We now consider the map $\Psi: Y \rightarrow V$ defined as

$$
\left.\Psi\right|_{L}:=f \circ g,\left.\quad \Psi\right|_{M}:=f,\left.\quad \Psi\right|_{-V}:=\mathrm{Id}_{-V}
$$

and we observe that

$$
\Psi \circ i_{1}=(f \circ g) \sqcup \operatorname{Id}_{(-V)}, \quad \Psi \circ i_{2}=f \sqcup(-f), \quad \Psi \circ j_{1}=f \circ\left(g \sqcup \operatorname{Id}_{(-M)}\right) .
$$

By applying $\tilde{\Psi}_{*}$ to both sides of 4.15 we then get, by functoriality, that

$$
\tilde{f}_{*}(\rho(g))+\rho(f)=\rho(f \circ g)+\tilde{f}_{*}\left(\rho\left(\operatorname{Id}_{M}\right)\right) \text { in } K_{n+1}\left(D^{*}(\widetilde{V})^{\Gamma}\right)
$$

which is precisely 4.14. Granted formula 4.14 we obtain, as in 25, Theorem 9.1]:

$$
\rho(a \cdot[M \stackrel{f}{\rightarrow} V])-\rho[M \stackrel{f}{\rightarrow} V]=\tilde{f}_{*}\left(\rho\left[\partial_{1} W \stackrel{\partial_{1} F}{\longrightarrow} M\right]\right)-\tilde{f}_{*}\left(\rho\left[\partial_{0} W \stackrel{\partial_{0} F}{\longrightarrow} M\right]\right) .
$$

By applying the delocalized APS index theorem 3.1 for perturbed operators and functoriality we recognize in the difference on the right hand side the element $i_{*} \operatorname{Ind}(a)$, with $\operatorname{Ind}(a)$ as in 4.13 . The commutativity of the first square is therefore established.

Next we tackle the commutativity of the second square, where we recall that the horizontal map $\mathcal{S}(V) \rightarrow$ $\mathcal{N}(V)$ is the forgetful map. Let $[M \stackrel{f}{\rightarrow} V] \in \mathcal{S}(V)$ and consider $Z=M \sqcup(-V)$ with the usual maps $\phi: Z \rightarrow V, \phi:=f \sqcup \operatorname{Id}_{(-V)}$, and $u_{Z}: Z \rightarrow B \Gamma, u_{Z}:=u \circ \phi$. We let $C_{f}$ be the trivializing perturbation defined by $f$, so that $D_{\widetilde{Z}}+C_{f}$ is invertible, with spectrum disjoint from the interval $[-2 \epsilon, 2 \epsilon]$. Recall that for any closed compact manifold $Z$ of odd dimension, endowed with a classifying map $u_{Z}: Z \rightarrow B \Gamma$,

$$
K_{1}(Z)=K_{0}\left(D^{*} Z / C^{*} Z\right)=K_{0}\left(D^{*}(\widetilde{Z})^{\Gamma} / C^{*}(\widetilde{Z})^{\Gamma}\right)
$$

with $\widetilde{Z}=u_{Z}^{*} E \Gamma$; in this realization of the K-homology groups, the K-homology signature class $\left[D_{Z}\right] \in K_{1}(Z)$ is obtained by considering the projection $\left[1 / 2\left(\chi\left(D_{\widetilde{Z}}\right)+1\right)\right]$ in $K_{0}\left(D^{*}(\widetilde{Z})^{\Gamma} / C^{*}(\widetilde{Z})^{\Gamma}\right)$ with $\chi$ a chopping function. The following continuous path of operators

$$
[0,1] \ni t \rightarrow\left[\frac{1}{2}\left(\chi\left(D_{\widetilde{Z}}+t C_{f}\right)+1\right)\right] \in K_{0}\left(D^{*}(\widetilde{Z})^{\Gamma} / C^{*}(\widetilde{Z})^{\Gamma}\right)
$$

shows that for a chopping function $\chi$ equal to 1 on $[\epsilon,+\infty)$ and -1 on $(-\infty, \epsilon]$ we have the equality

$$
\left[\frac{1}{2}\left(\frac{D_{\widetilde{Z}}+C_{f}}{\left|D_{\widetilde{Z}}+C_{f}\right|}+1\right)\right]=\left[1 / 2\left(\chi\left(D_{\widetilde{Z}}\right)+1\right)\right] \text { in } K_{0}\left(D^{*}(\widetilde{Z})^{\Gamma} / C^{*}(\widetilde{Z})^{\Gamma}\right) .
$$

With $\phi=f \sqcup \operatorname{Id}_{(-V)}$ we now apply $(\tilde{\phi})_{*}$ to both sides of this equality. On the left hand side we obtain the class $\rho[M \stackrel{f}{\rightarrow} V] \in K_{0}\left(D^{*}(\widetilde{V})^{\Gamma} / C^{*}(\widetilde{V})^{\Gamma}\right)=K_{1}(V)$ whereas on the right we obtain $\phi_{*}\left[D_{Z}\right] \in K_{1}(V)$ which is precisely $f_{*}\left[D_{M}\right]-\left[D_{V}\right]$. The commutativity of the second square is established.

Finally, we tackle the third square. The image of an element $[M \stackrel{f}{\rightarrow} V] \in \mathcal{N}(V)$ in $L_{n}(\mathbb{Z} \Gamma)$ is simply the class associated to the cycle represented by the closed odd dimensional manifold $Z=M \cup(-V)$ endowed with the classifying map $u_{Z}$. This element in $L_{n}(\mathbb{Z} \Gamma)$ is the surgery obstruction associated to $[M \stackrel{f}{\rightarrow} V] \in \mathcal{N}(V)$ and one of the fundamental results in surgery theory states that a normal map $f: M \rightarrow V$ can be surgered to a homotopy equivalence if and only if its surgery obstruction in $L_{n}(\mathbb{Z} \Gamma)$ vanishes. This is the meaning of exactness of the surgery sequence at $\mathcal{N}(V)$. Going back to the commutativity of the third square we observe that the map Ind sends this class, the surgery obstruction associated to $[M \stackrel{f}{\rightarrow} V]$, to the associated index class in $K_{n}\left(C_{r}^{*} \Gamma\right)$. By [6, Theorem 2], this class is equal to the Mishchenko symmetric signature of the covering $u_{Z}^{*} E \Gamma \rightarrow Z \equiv M \cup(-V)$. This remark reduces the proof of the commutativity of the third square to the proof given by Higson and Roe in [4, Theorem 5.4]. 
4.18 Remark. In [28, a slightly different approach to the delocalized APS index theorem for spin Dirac operators is worked out. It uses the localization algebras invented by $\mathrm{Yu}$ in [29], which allow to formulate and prove product formulas for products between fundamental classes (primary invariants) and rho-classes (secondary invariants). Presumably this approach can be generalized to the perturbed operators we have to use here; however, it is clear that one will face technical difficulties related to the non-locality of the perturbed operators and the very definition of localization algebras (based on locality of the operators involved).

4.19 Remark. So far we have treated only the case in which the manifold $V$ appearing in the structure set $\mathcal{S}^{h}(V)$ is odd dimensional. Similarly, in [16] we have only treated the group $\operatorname{Pos}_{n}^{\operatorname{spin}}(X)$ for $n$ odd. The problem, both here and in [16, is centered around the delocalized APS index theorem, a crucial tool which we prove only for even dimensional manifolds with boundary.

However, there is a clear strategy to deduce the remaining case from the one we have established, which is also suggested in 28. One should develop a "suspension homomorphism", mapping in functorial and compatible ways (for manifolds $W$ with and without boundary) $K_{*}\left(C^{*}(W)^{\Gamma}\right)$ to $K_{*+1}\left(C^{*}(W \times \mathbb{R})^{\Gamma \times \mathbb{Z}}\right)$, and $K_{*}\left(D^{*}(W)^{\Gamma}\right)$ to $K_{*+1}\left(D^{*}(W \times \mathbb{R})^{\Gamma \times \mathbb{Z}}\right)$.

These homomorphisms have to map the rho-classes (in the case of a manifold without boundary) and index classes (in general) of the Dirac operators on $W$ to those on $W \times \mathbb{R}$, and they have to be injective. It is then an easy exercise to deduce the delocalized APS index theorem for one parity from the one for the other parity.

This program has now been implemented by Vito Felice Zenobi in 31, building on previous work of Paul Siegel, see 21] and 22. Consequently, the delocalised APS index theorem presented in this article is now established in every dimension. Similarly, Zenobi shows that the delocalised APS index theorem proved in [16] is valid in all dimensions. The latter is also a consequence of the treatment given by Xie and Yu in 28].

Notice that a general treatment of the suspension homomorphism and the relevant compatibilities and product formulas, using Yu's localization algebras, is carried out by Rudolf Zeidler in [30, making more explicit some of the constructions in [28]. The methods in [30] also cover the case of the signature operator and its rho invariants and are therefore another route to derive the delocalzed APS index theorem for odd dimensional manifolds from the one for even dimensional manifolds.

\section{Proof of the delocalized APS index theorem}

In this section we prove Theorem 3.1. the delocalized APS index theorem for perturbed operators. Recall from [16, Section 4] that the corresponding result for the Dirac operator of a spin manifold with positive scalar curvature on the boundary is proved in two steps: first we show how to reduce the validity of the theorem on $\widetilde{W}_{\infty}$ to a version of the theorem on $\mathbb{R} \times \partial \widetilde{W}$ (which we call the "cylinder delocalized index theorem for perturbed operators"); next a detailed analysis, with explicit computations, is carried out on the cylinder in order to establish the cylinder delocalized index theorem. We will follow this strategy also here.

In this subsection we make more precise the notation given in the proof of Proposition 2.27, thus we denote by $p$ the characteristic function of $[0, \infty) \times \partial \widetilde{W}$ inside $W_{\infty}$ and we denote by $P$ the corresponding multiplication operator. Finally, as in the proof of Proposition 2.27, we denote by $p_{0}$ the characteristic function of $[0, \infty) \times \partial \widetilde{W}$ inside the full cylinder $\mathbb{R} \times \partial \widetilde{W}$ and by $P_{0}$ the corresponding multiplication operator.

\section{$5.1 \quad$ Reduction to the cylinder}

Once we have at our disposal Proposition 2.27, together with its proof, it is elementary to check that the reduction to the cylinder proceeds exactly as in [16]. Thus

(1) We establish that $P_{0} \chi(\bar{D}+\bar{C})_{+} P_{0}$ is an involution in $D^{*}([0, \infty) \times \partial \widetilde{W})^{\Gamma} / D^{*}(\{0\} \times \partial \widetilde{W} \subset[0, \infty) \times$ $\partial \widetilde{W})^{\Gamma}$, where we recall that on the cylinder the bundle on which the signature operator acts can be identified with the direct sum of two copies of the same bundle, with the obvious grading. We obtain $\partial\left[P_{0} \chi(\bar{D}+\bar{C})_{+} P_{0}\right] \in K_{0}\left(D^{*}(\partial \widetilde{W} \subset[0, \infty) \times \partial \widetilde{W})^{\Gamma}\right)$. 
(2) Similarly, we have a class $\partial[P \chi(\bar{D}+\bar{C}) P] \in K_{0}\left(D^{*}\left(\widetilde{W} \subset \widetilde{W}_{\infty}\right)^{\Gamma}\right)$, where it is obvious how to consider $P \chi(\bar{D}+\bar{C}) P$ as an element of $D^{*}\left(\widetilde{W}_{\infty}\right)^{\Gamma}$.

(3) Next we establish that

$$
\chi(D+C)-P \chi(\bar{D}+\bar{C}) P \in D^{*}\left(\widetilde{W} \subset \widetilde{W}_{\infty}\right)^{\Gamma}
$$

We shall prove (5.1) at the end of this subsection, just below.

(4) Using the latter information, we show that if $\iota_{*}: K_{*}\left(C^{*}\left(\widetilde{W} \subset \widetilde{W}_{\infty}\right)^{\Gamma}\right) \rightarrow K_{*}\left(D^{*}\left(\widetilde{W} \subset \widetilde{W}_{\infty}\right)^{\Gamma}\right)$ is induced by the inclusion, then $\iota_{*}\left(\operatorname{Ind}^{\mathrm{rel}}(D+C)\right)=\partial\left[P \chi(\bar{D}+\bar{C})_{+} P\right] \in K_{0}\left(D^{*}\left(\widetilde{W} \subset \widetilde{W}_{\infty}\right)^{\Gamma}\right)$.

(5) We then show that if $j_{+}$is the homomorphism $K_{*}\left(D^{*}(\partial \widetilde{W} \subset[0, \infty) \times \partial \widetilde{W})^{\Gamma}\right) \rightarrow K_{0}\left(D^{*}(\widetilde{W} \subset \widetilde{W} \infty)^{\Gamma}\right)$, then $j_{+}\left(\partial\left[P_{0} \chi(\bar{D}+\bar{C})_{+} P_{0}\right]\right)=\partial\left[P \chi(\bar{D}+\bar{C})_{+} P\right]$ in $K_{0}\left(D^{*}\left(\widetilde{W} \subset \widetilde{W}_{\infty}\right)^{\Gamma}\right)$.

(6) Next we assume the

5.2 Theorem (Cylinder delocalized index theorem for perturbed operators). If $j_{\partial}$ is the isomorphism $K_{*}\left(D^{*}(\partial \widetilde{W})^{\Gamma}\right) \rightarrow K_{*}\left(D^{*}(\partial \widetilde{W} \subset[0, \infty) \times \partial \widetilde{W})^{\Gamma}\right)$, then

$$
\partial\left[P_{0} \chi(\bar{D}+\bar{C})_{+} P_{0}\right]=j_{\partial} \rho\left(D_{\partial}+C_{\partial}\right) .
$$

(7) Finally we recall the following commutative diagram from [16, Proposition 3.1]:

$$
\begin{array}{ccc}
K_{*}\left(D^{*}(\partial \widetilde{W})^{\Gamma}\right) & \stackrel{j_{*}}{\longrightarrow} \quad K_{*}\left(D^{*}(\widetilde{W})^{\Gamma}\right) \\
\cong\left\lfloor j_{\partial}\right. & \cong \downarrow c \\
K_{*}\left(D^{*}(\partial \widetilde{W} \subset[0, \infty) \times \partial \widetilde{W})^{\Gamma}\right) \stackrel{j_{+}}{\longrightarrow} K_{*}\left(D^{*}\left(\widetilde{W} \subset \widetilde{W}_{\infty}\right)^{\Gamma}\right)
\end{array}
$$

Granted these properties one gets

$$
\iota_{*}\left(\operatorname{Ind}^{\mathrm{rel}}(D+C)\right)=\partial\left[P \chi(\bar{D}+\bar{C})_{+} P\right]=j_{+}\left(\partial\left[P_{0} \chi(\bar{D}+\bar{C})_{+} P_{0}\right]\right)=j_{+}\left(j_{\partial} \rho\left(D_{\partial}+C\right)\right) .
$$

Once we apply $c^{-1}$ to both sides we obtain precisely the equality in the theorem, i.e. $\iota_{*}(\operatorname{Ind}(D, C))=$ $j_{*}\left(\rho\left(D_{\partial}+C_{\partial}\right)\right)$.

Proof of 5.1). We go back to the notation adopted in the proof of Proposition 2.27, thus with a small abuse of notation we don't distinguish between $P_{0}$ and $P$. Recall that we identify the image of $P_{0}$ in $L^{2}(\mathbb{R} \times \widetilde{W})$ with the corresponding subspace of $L^{2}\left(\widetilde{W}_{\infty}\right)$ (in order to lighten the notation we do not write the vector bundles in the $L^{2}$-spaces). This way, we consider $P_{0} \chi(\bar{D}+\bar{C}) P_{0}$ as an operator on $L^{2}\left(\widetilde{W}_{\infty}\right)$.

First of all we remark that thanks to Proposition 2.27 we only need to establish (5.1) for one specific chopping function; we therefore choose $\chi(x)=x / \sqrt{1+x^{2}}$. We know, see [16. Lemma 4.12], that $\chi(D)-$ $P_{0} \chi(\bar{D}) P_{0} \in D^{*}\left(\widetilde{W} \subset \widetilde{W}_{\infty}\right)^{\Gamma}$. We also know from [2, Lemma 5.8] that

$$
\chi(\bar{D})-\chi(\bar{D}+\bar{C}) \in C^{*}(\mathbb{R} \times \partial \widetilde{W})^{\Gamma} ; \quad \chi(D)-\chi(D+C) \in C^{*}\left(\widetilde{W}_{\infty}\right)^{\Gamma} .
$$

Then we write

$$
\chi(D+C)-P_{0} \chi(\bar{D}+\bar{C}) P_{0}=(\chi(D+C)-\chi(D))+\left(\chi(D)-P_{0} \chi(\bar{D}) P_{0}\right)+\left(P_{0} \chi(\bar{D}) P_{0}-P_{0} \chi(\bar{D}+\bar{C}) P_{0}\right) .
$$

As already remarked, the second summand on the right hand side is an element in $D^{*}\left(\widetilde{W} \subset \widetilde{W}_{\infty}\right)^{\Gamma}$. We will prove that the sum of the first and third term on the right-hand side is in $C^{*}\left(\widetilde{W} \subset \widetilde{W}_{\infty}\right)^{\Gamma}$. To this end we 
write an explicit expression for this sum, using that $\chi(x)=x / \sqrt{1+x^{2}}$. Indeed, using once again [2, Lemma $5.8]$, we can write this sum as operator norm convergent integral

$$
\begin{aligned}
\frac{1}{\pi} \int_{1}^{\infty} \frac{t}{\sqrt{t^{2}-1}}\left((D+i t)^{-1} C(D+C+i t)^{-1}-P_{0}(\bar{D}+i t)^{-1} \bar{C}(\bar{D}+\bar{C}+i t)^{-1} P_{0}\right) d t \\
+\frac{1}{\pi} \int_{1}^{\infty} \frac{t}{\sqrt{t^{2}-1}}\left((D-i t)^{-1} C(D+C-i t)^{-1}-P_{0}(\bar{D}-i t)^{-1} \bar{C}(\bar{D}+\bar{C}-i t)^{-1} P_{0}\right) d t
\end{aligned}
$$

Therefore it suffices to show that the integrands are in $C^{*}\left(\widetilde{W} \subset \widetilde{W}_{\infty}\right)^{\Gamma}$. Let us consider the first integral and the t-dependent operator there:

$$
(D+i t)^{-1} C(D+C+i t)^{-1}-P_{0}(\bar{D}+i t)^{-1} \bar{C}(\bar{D}+\bar{C}+i t)^{-1} P_{0}
$$

Writing $1=P_{0}+\left(1-P_{0}\right)$ and reasoning as we did at the end of the proof of Proposition 2.27 (after the proof of Lemma 2.29, we see that $(5.5)$ is equal, up to a term in $C^{*}\left(\widetilde{W} \subset \widetilde{W}_{\infty}\right)^{\Gamma}$, to

$$
\begin{aligned}
P_{0}(D+i t)^{-1} P_{0} C P_{0}(D+C+i t)^{-1} P_{0}-P_{0}(\bar{D}+i t)^{-1} P_{0} \bar{C} P_{0}(\bar{D}+\bar{C}+i t)^{-1} P_{0} \\
=P_{0}(D+i t)^{-1} P_{0} C P_{0}\left(P_{0}(D+C+i t)^{-1} P_{0}-P_{0}(\bar{D}+\bar{C}+i t)^{-1} P_{0}\right)+ \\
\left(P_{0}(D+i t)^{-1} P_{0}-P_{0}(\bar{D}+i t)^{-1} P_{0}\right) P_{0} C P_{0}(\bar{D}+\bar{C}+i t)^{-1} P_{0}
\end{aligned}
$$

since, by definition, $P_{0} \bar{C} P_{0}=P_{0} C P_{0}$. We already know that $\left(P_{0}(D+C+i t)^{-1} P_{0}-P_{0}(\bar{D}+\bar{C}+i t)^{-1} P_{0}\right)$ and $\left(P_{0}(D+i t)^{-1} P_{0}-P_{0}(\bar{D}+i t)^{-1} P_{0}\right)$ are elements in $C^{*}\left(\widetilde{W} \subset \widetilde{W}_{\infty}\right)^{\Gamma}$. Since the other factors are all norm limits of finite propagation operators, thus multipliers of $C^{*}\left(\widetilde{W} \subset \widetilde{W}_{\infty}\right)^{\Gamma}$, we see that (5.5) is in $C^{*}\left(\widetilde{W} \subset \widetilde{W}_{\infty}\right)^{\Gamma}$. The proof of $(5.1)$ is complete.

\subsection{Proof of the cylinder delocalized index theorem for perturbed operators}

We now show Theorem 5.2 , namely that if $j_{\partial}$ is the isomorphism $K_{*}\left(D^{*}(\partial \widetilde{W})^{\Gamma}\right) \rightarrow K_{*}\left(D^{*}\left(\partial \widetilde{W} \subset \mathbb{R}_{\geq} \times\right.\right.$ $\left.\partial \widetilde{W})^{\Gamma}\right)$ induced by the inclusion, then $\partial\left[P_{0} \chi(\bar{D}+\bar{C})_{+} P_{0}\right]=j_{\partial} \rho\left(D_{\partial}+C_{\partial}\right)$ in $\quad K_{*}\left(D^{*}\left(\partial \widetilde{W} \subset \mathbb{R}_{\geq} \times \partial \widetilde{W}\right)^{\Gamma}\right)$, where we abbreviate $\mathbb{R}_{\geq}:=[0, \infty)$.

We wish to point out that our arguments, although somewhat lengthy, are elementary. It is certainly possible to envisage a proof based on the Volterra expansion for the wave operator of a perturbed Dirac operator. However, doing this properly does require some non-trivial work and, in addition, it would not generalize easily to Lipschitz manifolds. This is why we have followed the route presented below.

5.6 Notation. We set $\widetilde{M}:=\partial \widetilde{W}$. We denote the Dirac operator on $\widetilde{M}$, the cross-section of the cylinder, by $D$ and the Dirac operator on $\mathbb{R} \times \widetilde{M}$ by $\bar{D}$. We denote a trivializing perturbation for $D$ as $C$ and we denote the resulting perturbation on $\mathbb{R} \times \widetilde{M}$, the one obtained by extending $C$ to be constant in the $\mathbb{R}$-direction, by $\bar{C}$. We use the symbol $\mathcal{L}^{2}(\widetilde{M})$ for the $L^{2}$-sections of the relevant Clifford bundle. Similarly we employ $\mathcal{L}^{2}(\mathbb{R} \times \widetilde{M})$ and $\mathcal{L}^{2}\left(\mathbb{R}_{\geq} \times \widetilde{M}\right)$.

5.7 Proposition. The bounded linear operator

$$
V: \mathcal{L}^{2}(\widetilde{M}) \rightarrow \mathcal{L}^{2}([0, \infty) \times \widetilde{M}), \quad s \mapsto V s,(V s)(t):=\sqrt{2|D+C|} e^{-t|D+C|}(s)
$$

covers in the $D^{*}$-sense the inclusion $i: M \hookrightarrow \mathbb{R}_{\geq} \times M, m \rightarrow(0, m)$.

Proof. We prove this in Section 5.2.1.

5.9 Proposition. The operator $\frac{|D+C|+\partial_{t}}{(D+C)-\partial_{t}}$ belongs to $D^{*}(\mathbb{R} \times \widetilde{M})^{\Gamma}$.

Proof. We prove this in Section 5.2 .2 . 
Granted these two Propositions, we can proceed as in [16, Section 4.4]. We have the following proposition.

5.10 Proposition. Set $Q:=P_{0} \frac{|D+C|-\partial_{t}}{(D+C)+\partial_{t}} P_{0}: \mathcal{L}^{2}\left(\mathbb{R}_{\geq} \times \widetilde{M}\right) \rightarrow \mathcal{L}^{2}\left(\mathbb{R}_{\geq} \times \widetilde{M}\right)$. Then

$$
\left(P_{0} \frac{|D+C|+\partial_{t}}{(D+C)-\partial_{t}} P_{0}\right) \circ Q=\operatorname{Id}_{\mathcal{L}^{2}\left(\mathbb{R}_{\geq} \times \widetilde{M}\right)} ; \quad Q \circ\left(P_{0} \frac{|D+C|+\partial_{t}}{(D+C)-\partial_{t}} P_{0}\right)=\operatorname{Id}_{\mathcal{L}^{2}\left(\mathbb{R}_{\geq} \times \widetilde{M}\right)}-\Pi
$$

with $\Pi:=V \chi_{[0, \infty)}(D+C) V^{*}$.

This implies that

$$
\partial\left[P_{0} \frac{|D+C|+\partial_{t}}{(D+C)-\partial_{t}} P_{0}\right]=\left[V \chi_{[0, \infty)}(D+C) V^{*}\right] \quad \text { in } \quad K_{0}\left(D^{*}\left(\widetilde{M} \subset \mathbb{R}_{\geq} \times \widetilde{M}\right)^{\Gamma}\right) .
$$

The proof of Proposition 5.10 proceeds as in [16, Section 4.4], given that the arguments there are purely functional analytic and rest ultimately on the Browder-Garding decomposition for the self-adjoint operator $D+C$. Now, on the right hand side of Equation (5.12) we have, by definition, $j_{\partial} \rho\left(D_{\partial}+C_{\partial}\right)$ whereas the left hand side is equal to $\partial\left[P_{0} \chi(\bar{D}+\bar{C})_{+} P_{0}\right]$. This assertion, namely that

$$
\partial\left[P_{0} \frac{|D+C|+\partial_{t}}{(D+C)-\partial_{t}} P_{0}\right]=\partial\left[P_{0} \chi(\bar{D}+\bar{C})_{+} P_{0}\right]
$$

is proved as in [16], using exactly the deformation argument explained there, after the proof of Proposition 4.33. Thus, assuming Proposition 5.7 and Proposition 5.9, we have proved the cylinder delocalized index theorem 5.2 .

\subsubsection{Proof of Proposition 5.7; the map $V$ covers the inclusion in the $D^{*}$-sense}

We recall first of all that, by definition, $V: \mathcal{L}^{2}(\widetilde{M}) \rightarrow \mathcal{L}^{2}([0, \infty) \times \widetilde{M})$ covers in the $D^{*}$-sense the inclusion $i: \widetilde{M} \hookrightarrow \mathbb{R}_{\geq} \times \widetilde{M}$ if $V$ is the norm-limit of bounded $\Gamma$-equivariant maps $U$ satisfying the following two conditions:

- there is an $R>0$ such that $\phi U \psi=0$ if $d(\operatorname{Supp}(\phi), i(\operatorname{Supp}(\psi)))>R$, for $\phi \in C_{c}\left(\mathbb{R}_{\geq} \times \widetilde{M}\right)$ and $\psi \in C_{c}(\widetilde{M})$

- $\phi U \psi$ is compact for each $\phi \in C_{0}\left(\mathbb{R}_{\geq} \times \widetilde{M}\right)$ and $\psi \in C_{c}(\widetilde{M})$ such that $\operatorname{Supp}(\phi) \cap i(\operatorname{Supp}(\psi))=\emptyset$

5.14 Notation. In all this subsection we shall denote by $M$ the total space of a Riemannian $\Gamma$-Galois covering with compact base $M / \Gamma$. As before, we write $D$ for a Dirac type operator on $M$, acting on sections of a Clifford module bundle. We will in the notation ignore this bundle.

Let $E: L^{2}(M) \rightarrow L^{2}(M)$ be a bounded equivariant self-adjoint operator, norm limit of equivariant finite propagation operators and with the property that $D+E$ is invertible.

Recall that we set $V: L^{2}(M) \rightarrow L^{2}([0, \infty) \times M)=L^{2}\left([0, \infty), L^{2}(M)\right) ;(V u)(t)=\sqrt{2|D+E|} e^{-t|D+E|} u$.

5.15 Lemma. Let $H$ be a Hilbert space and $W: H \rightarrow L^{2}([0, \infty), H)$ be an operator of the form $W(u)(t)=$ $f_{t}(A) u$ for a self-adjoint operator $A$ on $H$ and a measurable function $[0, \infty) \rightarrow C_{b}(\mathbb{R}) ; t \mapsto f_{t}$.

Then $\|W\|^{2} \leq \sup _{\lambda \in \operatorname{Spec}(A)} \int_{0}^{\infty}\left|f_{t}(\lambda)\right|^{2} d t$.

Proof. By definition, for $u \in H$ we have $|W u|^{2}=\int_{0}^{\infty}\left|f_{t}(A) u\right|_{H}^{2} d t$. Use the Brower-Garding decomposition as direct integral $H=\int H_{\lambda} d \mu(\lambda)$ according to the spectral decomposition of the self-adjoint operator $A$. 
Then $u=\int_{\operatorname{Spec}(A)} u_{\lambda} d \mu(\lambda)$ with $u_{\lambda} \in H_{\lambda}$ and $|u|_{H}^{2}=\int\left|u_{\lambda}\right|_{H_{\lambda}}^{2} d \mu(\lambda)$. Moreover, for a function $f(\lambda)$ we have $f(A) u=\int_{\operatorname{Spec}(A)} f(\lambda) u_{\lambda} d \mu(\lambda)$. Then

$$
\begin{aligned}
|W u|^{2} & =\int_{0}^{\infty}\left|f_{t}(A) u\right|^{2} d t=\int_{0}^{\infty} \int_{\operatorname{Spec}(A)}\left|f_{t}(\lambda) u_{\lambda}\right|_{H_{\lambda}}^{2} d \mu(\lambda) d t \\
& \stackrel{\text { Fubini }}{=} \int_{\operatorname{Spec}(A)} \int_{0}^{\infty}\left|f_{t}(\lambda)\right|^{2} d t\left|u_{\lambda}\right|_{H_{\lambda}}^{2} d \mu(\lambda) \leq\left(\sup _{\lambda \in \operatorname{Spec}(A)} \int_{0}^{\infty}\left|f_{t}(\lambda)\right|^{2} d t\right) \cdot \underbrace{\int_{\operatorname{Spec}(A)}\left|u_{\lambda}\right|_{H_{\lambda}}^{2} d \mu(\lambda)}_{=|u|_{H}^{2}} .
\end{aligned}
$$

5.16 Lemma. The operator $P_{R} V: L^{2}(M) \rightarrow L^{2}([0, \infty) \times M)$ converges to 0 in norm for $R \rightarrow \infty$, where $P_{R}$ is the orthogonal projection onto $L^{2}([R, \infty) \times M)$

Proof. By assumption, $D+E$ is invertible, i.e. there is $\epsilon>0$ such that $(-\epsilon, \epsilon) \cap \operatorname{Spec}(D+E)=\emptyset$. We can write $P_{R} \circ V(u)(t)=f_{t}^{R}(D+E) u$ with $f_{t}^{R}(\lambda)=\left\{\begin{array}{ll}0 ; & t>R \\ \sqrt{2|\lambda|} \exp (-t|\lambda|) ; & t \leq R\end{array}\right.$. For $|\lambda| \geq \epsilon$, the $L^{2}$-norm of $t \mapsto f_{t}^{R}(\lambda)$ uniformly tends to 0 as $R \rightarrow \infty$. By Lemma $5.15 .\left\|P_{R} V\right\| \stackrel{R \rightarrow \infty}{\longrightarrow} 0$.

We now treat $\left(1-P_{R}\right) V$. Define $V_{D}: L^{2}(M) \rightarrow L^{2}([0, \infty) \times M)$ by $V_{D} u(t)=\sqrt{2|D|} e^{-t|D|} u$ i.e. exactly as $V$, but with $D+E$ replaced by $D$. We want to prove the properties for $V$ by a comparison with $V_{D}$, where corresponding properties have been established in 16. For the comparison we use a resolvent trick, which requires some preparation.

5.17 Lemma. The function $\sqrt{|\lambda|} e^{-|\lambda|}$ can on $\mathbb{R}$ be approximated in supremum norm by $f(\lambda)$ which is a polynomial in $1 /\left(\lambda^{2}+1\right)$ (without constant term, i.e. vanishing at infinity) in such a way that even $e^{-|\lambda|}$ is approximated in $L^{2}(\mathbb{R})$ by $f(\lambda) / \sqrt{|\lambda|}$.

Proof. Consider the subalgebra of $C_{0}(\mathbb{R})$ generated by $w(t):=1 /\left(1+t^{2}\right)$ and $v(t):=t /\left(1+t^{2}\right)$ which is a subalgebra that separates points. Notice the relation

$$
v^{2}=w-w^{2} .
$$

Observe that the image of this subalgebra under the operator of differentiation contains for $n \geq 1$ the function $w^{n} v$; indeed $w^{\prime}=-2 w v$, so that $\left(w^{n}\right)^{\prime}=-2 n w^{n} v$ for all $n \geq 1$.

Consider now the subalgebra of $C_{0}(\mathbb{R})$ generated by $w$ and $w v$. This subalgebra also separates points; thus, by Stone-Weiestrass, every function in $C_{0}(\mathbb{R})$ can be approximated by a polynomial $P(w, w v)$ with $P(0,0)=0$ (elements in $C_{0}(\mathbb{R})$ vanish at infinity). Because of $(5.18$ we see that any continuous even function in $C_{0}(\mathbb{R})$ can be approximated by a polynomial in $w$ without constant term, whereas any continuous odd function in $C_{0}(\mathbb{R})$ can be approximated by a polynomial of the form $P(w) v$, with $P(0)=0$. Notice, finally, that because of the remark following (5.18) the antiderivative of a function of the form $P(w) v$, $P(0)=0$ belongs to the algebra generated by $w$ and $v$. Given $\epsilon>0$, we apply all this to $h^{\prime}(t)$, with $h(t):=u(t) e^{-|t|}\left(1+t^{2}\right)$ and $u(t):=\left\{\begin{array}{ll}\sqrt{|t|} ; & |t| \geq \epsilon \\ \frac{7}{4} \epsilon^{-3 / 2} t^{2}-\frac{3}{4} \epsilon^{-7 / 2} t^{4} ; & |t| \leq \epsilon\end{array}\right.$.

Note that $\left|u(t) e^{-|t|}-\sqrt{|t|} e^{-|t|}\right| \leq 4 \sqrt{\epsilon}$.

As $h$ is even and continuously differentiable, $h^{\prime}$ is odd and continuous. Because of the exponential decay of $e^{-|t|}, h^{\prime}(t)$ vanishes at $\infty$. Therefore, we can find an odd function $Q_{\epsilon}(t)=R(w(t)) v(t)$, with $R$ a polynomial without constant term, such that $\left|h^{\prime}(t)-Q_{\epsilon}(t)\right|<\epsilon$ for all $t \in \mathbb{R}$.

Then the even function $P_{\epsilon}(t):=\int_{0}^{t} Q_{\epsilon}(\tau) d \tau$ is a polynomial without constant term in $w(t)=1 /\left(1+t^{2}\right)$ such that $\left|h(t)-P_{\epsilon}(t)\right| \leq \epsilon|t|$ for all $t \in \mathbb{R}$, and therefore we have 


$$
\left|u(t) e^{-|t|}-\frac{P_{\epsilon}(t)}{\left(1+t^{2}\right)}\right| \leq \epsilon \frac{|t|}{1+t^{2}} \Longrightarrow\left|u(t) e^{-|t|}-\frac{P_{\epsilon}(t)}{1+t^{2}}\right| \leq \epsilon \cdot C .
$$

This shows that $\left(1+t^{2}\right)^{-1} P_{\epsilon}(t)$ approximates $\sqrt{|t|} e^{-|t|}$ in supremum norm.

Finally,

$$
\begin{aligned}
& \left|\frac{u(t)}{\sqrt{|t|}} e^{-|t|}-\frac{P_{\epsilon}(t)}{\sqrt{|t|}\left(1+t^{2}\right)}\right|_{L^{2}(\mathbb{R})} \leq \epsilon\left(\int_{-\infty}^{\infty} \frac{|t|}{\left(1+t^{2}\right)^{2}} d t\right)^{1 / 2} \leq \epsilon C, \\
& \left|\frac{\left|u(t) e^{-|t|}\right|}{\sqrt{|t|}}-e^{-|t|}\right|_{L^{2}(\mathbb{R})} \leq\left|\frac{7}{4} \epsilon^{-3 / 2} t^{2}-\frac{3}{4} \epsilon^{-7 / 4} t^{4}-1\right|_{L^{2}([-\epsilon, \epsilon])} \\
& \leq\left(\int_{-\epsilon}^{\epsilon}\left|\frac{7}{4} \frac{1}{\epsilon^{3 / 2}} t^{2}\right|^{2} d t\right)^{1 / 2}+\left(\int_{-\epsilon}^{\epsilon}\left|\frac{3}{4} \frac{1}{\epsilon^{7 / 2}} t^{4}\right|^{2} d t\right)^{1 / 2}+\left(\int_{-\epsilon}^{\epsilon} 1 d t\right)^{1 / 2} \leq C \epsilon
\end{aligned}
$$

with a constant $C$ independent of $\epsilon$.

5.19 Definition. For $f: \mathbb{R} \rightarrow \mathbb{R}$ continuous and sufficiently decaying at $\infty$, define

$$
V_{f}: L^{2}(M) \rightarrow L^{2}([0, \infty) \times M) \quad \text { by } \quad V_{f} u(t):=\frac{1}{\sqrt{|t|}} f(t|D+E|) u,
$$

and correspondingly $V_{f, D}$ with $D+E$ replaced by $D$.

5.20 Lemma. In the situation above, $\max \left\{\left\|V_{D}-V_{f, D}\right\|^{2},\left\|V-V_{f}\right\|^{2}\right\} \leq \int_{0}^{\infty}\left|\frac{f(t)}{\sqrt{t}}-e^{-t}\right|^{2} d t$.

Proof. By definition, $\left(V-V_{f}\right) u(t)=g_{t}(D+E) u$ with $g_{t}(\lambda)=\frac{\sqrt{2|t \lambda|} e^{-t|\lambda|}-f(t|\lambda|)}{\sqrt{t}}$. Then

$$
\int_{0}^{\infty}\left|g_{t}(\lambda)\right|^{2} d t=\int_{0}^{\infty}\left|\frac{1}{\sqrt{t}}\left(\sqrt{2|t \lambda|} e^{-t|\lambda|}-f(t|\lambda|)\right)\right|^{2} d t \stackrel{s=t|\lambda|}{=} \int_{0}^{\infty} \frac{1}{s}\left|\sqrt{s} e^{-s}-f(s)\right|^{2} d s .
$$

By Lemma 5.15, $\max \left\{\left\|V_{D}-V_{f, D}\right\|^{2},\left\|V-V_{f}\right\|^{2}\right\} \leq \int_{0}^{\infty}\left|g_{t}(\lambda)\right|^{2} d t=\int_{0}^{\infty}\left|e^{-t}-\frac{f(t)}{\sqrt{t}}\right|^{2} d t$, as claimed.

Because of this, in light of Lemma 5.15 we can find a polynomial $f(t)$ in $\left(1+t^{2}\right)^{-1}$, vanishing at $\infty$ such that $V-V_{f}$ and $V_{D}-V_{f, D}$ are arbitrarily small in norm. The same applies then of course also to $\left(1-P_{R}\right)\left(V-V_{f}\right)$ and $\left(1-P_{R}\right)\left(V_{D}-V_{f, D}\right)$.

5.21 Lemma. For a fixed polynomial $f(t)$ in $\left(1+t^{2}\right)^{-1}, V_{f}-V_{f, D}$ is a linear combination of operators of the form

$$
\begin{gathered}
u \mapsto\left(t \mapsto \frac{1}{\sqrt{|t|}} \frac{t(D+E)}{\left(1+t^{2}(D+E)^{2}\right)^{l}} t E \frac{1}{\left(1+t^{2} D^{2}\right)^{k}} u\right) \\
u \mapsto\left(t \mapsto \frac{1}{\sqrt{|t|}} \frac{1}{\left(1+t^{2}(D+E)^{2}\right)^{l}} t E \frac{t D}{\left(1+t^{2} D^{2}\right)^{k}} u\right)
\end{gathered}
$$

with $k, l \geq 1$.

Proof. First, expand $a^{n}-b^{n}=\sum_{k=1}^{n} a^{k-1}(a-b) b^{n-k}$. Apply this to $a=\left(1+t^{2}(D+E)^{2}\right)^{-1}$ and $b=$ $\left(1+t^{2} D^{2}\right)^{-1}$. Then

$$
a-b=\frac{1}{1+t^{2}(D+E)^{2}}\left(1+t^{2} D^{2}-1-t^{2}(D+E)^{2}\right) \frac{1}{1+t^{2} D^{2}}
$$


and $1+t^{2} D^{2}-1-t^{2}(D+E)^{2}=-t(D+E) t E-t E t D$.

Recall that for $g(t)=\frac{1}{\left(1+t^{2}\right)^{n}}$ we have $\left(\left(V_{g}-V_{g, D}\right) u\right)(t)=\frac{1}{\sqrt{|t|}}\left(\left(\frac{1}{1+t^{2}(D+E)^{2}}\right)^{n}-\left(\frac{1}{1+D^{2}}\right)^{n}\right) u$. Application of the formulas just derived immediately gives the result.

5.23 Lemma. If $f(t)$ is a polynomial in $\left(1+t^{2}\right)^{-1}$ then

$$
\lim _{r \rightarrow 0}\left\|\left(1-P_{r}\right)\left(V_{f}-V_{f, D}\right)\right\|=0 .
$$

Proof. Because of Lemma 5.21 it suffices to consider the operators given in 5.22 . Because of the factor $t$ in front of $t E$ which is always present, they are of the form

$$
u \mapsto(t \mapsto \sqrt{t} A(t) u)
$$

where $A(t)$ is a uniformly norm bounded family of operators: it is a composition of $E$ and of uniformly in $t$ operator norm bounded functions of $(D+E)$ and of $D$. The statement follows immediately from the definition of the norm on $L^{2}\left([0, \infty), L^{2}(M)\right)$.

5.24 Lemma. If $f(t)$ is a polynomial in $\left(1+t^{2}\right)^{-1}$ and $0<r<R<\infty$ then $\left(P_{r}-P_{R}\right)\left(V_{f}-V_{f, D}\right)$ is a norm limit of operators of finite propagation.

Proof. By Lemma 5.21, it suffices to compose the operators in $(5.22)$ with $\left(P_{r}-P_{R}\right)$ and prove the statement for these compositions. Now observe that these operators have the form $u \mapsto(t \mapsto A(t) u)$ where $A(t)=$ $\left\{\begin{array}{ll}0 ; & t>R, t<r \\ \phi_{t}(D+E) E \psi_{t}(D) ; & r \leq t \leq R\end{array}\right.$. Then $A(t)$ is a norm continuous function with values in operators which are norm limits of finite propagation operators: indeed, $\phi_{t}(\lambda), \psi_{t}(\lambda)$ tend to 0 for $\lambda \rightarrow \pm \infty$ and depend continuously in supremum norm on $t$, so that $\phi_{t}(D+E), \psi_{t}(D)$ are really limits of finite propagation operators and depend norm continuously on $t$. Therefore we can - up to an arbitrarily small error in norm - replace the function $A(t)$ by a (say piecewise constant) function $B(t)$ of operators with fixed finite propagation $S$. It follows that $u \mapsto(t \mapsto B(t) u)$ has finite propagation at $\operatorname{most} \max \{R, S\}$.

5.25 Lemma. If $f(t)$ is a polynomial in $\left(1+t^{2}\right)^{-1}$ and $0<r<R<\infty$ then $\left(P_{r}-P_{R}\right)\left(V_{f}-V_{f, D}\right)=$ $B(i+r D)^{-1}$ for a bounded operator $B$

Proof. Because of Lemma 5.21, we have to show that

$$
u \mapsto\left(t \mapsto\left\{\begin{array}{ll}
0 ; & t>R \text { or } t<r \\
\frac{(t(D+E))^{\eta}}{\left(1+t^{2}(D+E)^{2}\right)^{k}} \sqrt{|t|} E \frac{(t D)^{1-\eta}(i+r D)}{\left(1+t^{2} D^{2}\right)^{l}} u ; & r \leq t \leq R
\end{array}\right)\right.
$$

with $k, l \geq 1$ and $\eta \in\{0,1\}$ is bounded. Using that $\eta=0$ or $\eta=1$ and $l \geq 1$ this follows from the fact that $\lambda \mapsto \frac{r \lambda}{1+t^{2} \lambda^{2}}$ and $\lambda \mapsto \frac{t \lambda r \lambda}{1+t^{2} \lambda^{2}}$ are uniformly (in $t \geq r$ ) bounded functions of $\lambda$ (note that we substitute $D$ into these functions to obtain one factor making up the operator we have to consider, the remaining factors being controlled by the previous considerations).

5.26 Theorem. $V: L^{2}(M) \rightarrow L^{2}([0, \infty) \times M)$ covers the inclusion $\{0\} \times M \rightarrow[0, \infty) \times M$ in the $D^{*}$ sense.

Proof. Choose a polynomial $f(t)$ in $\left(1+t^{2}\right)^{-1}$ as above such that $\left(1-P_{R}\right)\left(V-V_{f}\right)$ and $\left(1-P_{R}\right)\left(V_{f, D}-V_{D}\right)$ have small norm for all $R>0$. We write

$V=P_{R} V+\left(1-P_{R}\right)\left(V-V_{f}\right)+\left(1-P_{r}\right)\left(V_{f}-V_{f, D}\right)+\left(P_{r}-P_{R}\right)\left(V_{f}-V_{f, D}\right)+\left(1-P_{R}\right)\left(V_{f, D}-V_{D}\right)+\left(1-P_{R}\right) V_{D}$.

for a suitable choice of $R$ such that, using Lemma 5.16, $P_{R} V$ has small norm, and of $r$ such that, using Lemma 5.23, $\left(1-P_{r}\right)\left(V_{f}-V_{f, D}\right)$ has small norm. 
By Lemma 5.24. $\left(P_{r}-P_{R}\right)\left(V_{f}-V_{f, D}\right)$ is a norm limit of finite propagation operators. Let $\phi$ be a compactly supported continuous function on $M$ and $\psi$ a compactly supported continuous function on $[0, \infty) \times M$ with $\operatorname{supp}(\psi) \cap\{0\} \times \operatorname{supp}(\phi)=\emptyset$. Then $(i+r D)^{-1} \phi: L^{2}(M) \rightarrow L^{2}(M)$ is compact and therefore, by Lemma 5.25 also $\psi\left(P_{r}-P_{R}\right)\left(V_{f}-V_{f, D}\right) \phi$ is compact (pseudolocal condition). Finally, in [16] we have shown, using unit propagation speed of the wave operator of $D$ on $M$ that $\left(1-P_{R}\right) V_{D}$ is a norm limit of finite propagation operators that satisfy, in addition, the pseudolocal condition. Note that these derivations did not use invertibility of $D$ and therefore are valid in the present context. Summarizing, we have shown that $V$ is a norm limit of finite propagation operators $F_{\epsilon}$ with the additional property that $\psi F_{\epsilon} \phi$ is compact for any $\phi \in C_{0}(M)$ and $\psi \in C_{0}([0, \infty) \times M)$ with $\operatorname{supp}(\psi) \cap\{0\} \times \operatorname{supp}(\phi)=\emptyset$. This proves that $V$ covers the inclusion in the $D^{*}$-sense.

The proof of Propositions 5.7 is now complete.

\subsubsection{Proof of Propositions 5.9 the operator $\frac{|D+C|+\partial_{t}}{D+C-\partial_{t}}$ belongs to $D^{*}(\widetilde{M} \times \mathbb{R})^{\Gamma}$}

5.27 Notation. In all this subsection we shall denote by $M$ the total space of a Riemannian $\Gamma$-Galois covering with compact base $M / \Gamma$. We also consider a Riemannian manifold $N$. (In the application we have in $\operatorname{mind} N=\mathbb{R}$.)

We consider a $\Gamma$-equivariant Dirac type operator $D$ on $M$ acting on the sections of a Clifford module bundle; as before, we will in the notation ignore this bundle. Similarly, we consider a Dirac type operator $\partial$ on $N$ (and in the applications we have in mind we shall in fact take $\partial=i \partial_{t}$ on $N=\mathbb{R}$ ). We recall that $D$ and $\partial$ are essentially self-adjoint; we shall not distinguish notationally between $D, \partial$ and their unique self-adjoint extensions.

We wish to prove that the operator $\frac{|D+C|+\partial_{t}}{D+C-\partial_{t}}$ belongs to $D^{*}(M \times \mathbb{R})^{\Gamma}$. In the course of the argument, it turns out that it is useful to work not only with $L^{2}$, but also with the Sobolev spaces $H^{1}, H^{2}$ on our complete manifold $M$, or $M \times N$. We have to understand mapping properties for perturbed Dirac operators and functions of those, acting on these Sobolev spaces. Because we don't want to assume that our perturbation is a pseudodifferential operator, we can't use standard mapping properties here; instead we will rely on abstract functional analysis of unbounded operators on Hilbert spaces.

5.28 Definition. For a manifold like $M, H^{1}(M)$ is defined as the domain of the (unique self-adjoint extension of the) Dirac type operator $D$ endowed with the graph norm $\left.\left(|D s|^{2}+|s|^{2}\right)\right)^{1 / 2}$. Similarly, $H^{2}(M)$ is the domain of $D^{2}$, endowed with the corresponding graph norm.

5.29 Proposition. Let $E$ be a self-adjoint bounded equivariant operator. Then $H^{1}(M)$ coincides with the domain of $D+E$ and its norm is equivalent to the graph norm $\left(|(D+E) s|^{2}+|s|^{2}\right)^{1 / 2}$. If $D+E$ is invertible, this norm is also equivalent to $|(D+E) s|$. In this case, $(D+E)^{-1}: L^{2} \rightarrow H^{1}$ is bounded, even an isometry.

Similarly, $H^{2}(M)$ is equal to the domain of $D^{2}+1$ endowed with the graph norm; in particular $\left(D^{2}+\right.$ $1): L^{2} \rightarrow H^{2}$ is an isometry for the appropriate choice of norm on $\mathrm{H}^{2}$.

Any suitably equivariant differential operator of order 1 is a bounded operator $H^{1} \rightarrow L^{2}$. In particular, on $M \times N$ both $D$ and $\partial$ are bounded operators $H^{1}(M \times N) \rightarrow L^{2}(M \times N)$.

Finally, $|D|$ and $|D+E|$ are bounded maps $H^{1} \rightarrow L^{2}$.

Proof. Only the statements about $|D|,|D+E|$ are not easy or standard. For those, we can write $|D+E|=$ $\frac{|D+E|}{D+E+i}(D+E+i)$, where the bounded function $\frac{|D+E|}{D+E+i}$ of $D+E$ is a bounded operator on $L^{2}$ and $D+E+i$ is bounded from $H^{1} \rightarrow L^{2}$.

5.30 Proposition. Given the Riemannian product $M \times N$, a Dirac type operator $D$ on $M$ with bounded equivariant self-adjoint perturbation $E: L^{2}(M) \rightarrow L^{2}(M)$, a compactly supported function $\phi$ on $N$ (acting by pointwise multiplication) and a compact operator $K: L^{2}(M) \rightarrow L^{2}(M)$, the composition

$$
H^{1}(M \times N) \stackrel{D+E}{\longrightarrow} L^{2}(M \times N)=L^{2}(M) \otimes L^{2}(N) \stackrel{K \otimes \phi}{\longrightarrow} L^{2}(M \times N)
$$


is compact.

Proof. Using the usual reduction techniques (write $\phi$ as a finite sum of functions with support in a coordinate neighborhood, use charts to plant these coordinate neighborhoods into $T^{l}$ ) one reduces to the case where $N=T^{l}$. Then, the operator with general $\phi$ is the composition of the bounded operator on $L^{2}\left(M \times T^{l}\right)$ given by multiplication with $\phi$ with the special operator where $\phi=1$. It therefore suffices to show that the latter one is compact, and we set $\phi=1$.

We now apply the strategy of the proof of the Rellich lemma. We have to understand a bit better the domain $H^{1}\left(M \times T^{l}\right)$ which, as the domain of the (perturbed) Dirac operator on $M \times T^{l}$ is a subspace of $L^{2}\left(M \times T^{l}\right)$ (with its own norm). We write $L^{2}\left(M \times T^{l}\right)=L^{2}(M) \otimes L^{2}\left(T^{l}\right)$. Using Fourier transform, we unitarily identify $L^{2}\left(T^{l}\right)$ with $l^{2}\left(\mathbb{Z}^{l}\right)$. Using the Browder-Garding spectral decomposition for the self-adjoint unbounded operator $D+E$, we write as a direct integral $L^{2}(M)=\int d \mu(\lambda) H_{\lambda}$. By the definition of compact operators as norm limits of finite rank operators, we can replace $K: L^{2}(M) \rightarrow L^{2}(M)$ up to an error of arbitrarily small norm by a finite rank operator $K_{\Lambda}$ such that $K_{\Lambda}$ maps $\int_{-\Lambda}^{\Lambda} H_{\lambda}$ to itself and is zero on the complement. By definition of the spectral decomposition, $D+E$ acts on the direct integral by multiplication with the spectral parameter $\lambda$. In particular, $\int_{-\Lambda}^{\Lambda} H_{\lambda}$ is entirely contained in the domain of $D+E$ (i.e. in $\left.H^{1}(M)\right)$ and restricted to this subspace the norm of $D+E$ is certainly bounded by the norm of $D+E$ as a bounded operator from $H^{1}$ to $L^{2}$. We denote this norm $C$.

Thus the Hilbert space $H^{1}\left(M \times T^{l}\right)$ has the direct summand $\int_{-\Lambda}^{\Lambda} H_{\lambda} \otimes H^{1}\left(T^{l}\right)$. Here, after Fourier transform $L^{2}\left(T^{l}\right) \cong l^{2}\left(\mathbb{Z}^{l}\right)$, we identify $H^{1}\left(T^{l}\right)$ with the domain of the operator

$$
l^{2}\left(\mathbb{Z}^{l}\right) \rightarrow l^{2}\left(\mathbb{Z}^{l}\right) ;\left(\lambda_{n}\right)_{n \in \mathbb{Z}^{k}} \mapsto\left(|n| \lambda_{n}\right)_{n \in \mathbb{Z}}, \quad \text { with }\left|n_{1}, \ldots, n_{l}\right|=\left|n_{1}\right|+\cdots+\left|n_{l}\right|
$$

endowed with the graph norm. For $R>0$, split

$$
H^{1}\left(T^{l}\right):=V_{R} \oplus V_{R}^{\perp} \quad \text { where } \quad V_{R}=\left\{\left(\lambda_{n}\right)_{n \in \mathbb{Z}^{l}} \mid \lambda_{n}=0 \text { if }|n|>R\right\} \text { is finite dimensional. }
$$

Note that the inclusion $V_{R}^{\perp} \hookrightarrow l^{2}\left(\mathbb{Z}^{l}\right)$ has norm $<R^{-1}$. We now conclude the following:

(1) The operator $\left(K \otimes \operatorname{id}_{L^{2}\left(T^{l}\right)}\right) \circ(D+E): H^{1}\left(M \times T^{k}\right) \rightarrow L^{2}(M) \otimes L^{2}\left(T^{k}\right)$ is norm close to $\left(K_{\Lambda} \otimes\right.$ $\left.\operatorname{id}_{L^{2}\left(T^{l}\right)}\right) \circ(D+E)$.

(2) restricted to the direct summand $\int_{-\Lambda}^{\Lambda} H_{\lambda} \otimes V_{R}$ of $H^{1}\left(M \times T^{l}\right),\left(K_{\Lambda} \otimes \mathrm{id}\right) \circ(D+E)$ has finite rank with image $\operatorname{im}\left(K_{\Lambda}\right) \otimes V_{R}$.

(3) restricted to the direct summand $\int_{-\Lambda}^{\Lambda} H_{\lambda} \otimes V_{R}^{\perp}$, the operator $D+E: H^{1}\left(M \times T^{k}\right) \rightarrow L^{2}\left(M \times T^{k}\right)$ has norm $\leq C \cdot R^{-1}$, where $R^{-1}$ comes from the ratio of the $H^{1}$-norm and the $L^{2}$-norm on $V_{R}^{\perp}$.

Finally, on the orthogonal complement of $\int_{-\Lambda}^{\Lambda} H_{\lambda} \otimes H^{1}\left(T^{k}\right)$ in $H^{1}\left(M \times T^{k}\right)$, by the choice of $K_{\Lambda}$, the operator $\left(K_{\Lambda} \otimes \mathrm{id}\right) \circ(D+E)$ vanishes.

(4) It follows that $\left(K_{\Lambda} \otimes \mathrm{id}\right) \circ(D+E)$ is up to an error of norm $C / R$ a finite rank operator.

(5) All together, $(K \otimes \mathrm{id}) \circ(D+E): H^{1}\left(M \times T^{l}\right) \rightarrow L^{2}\left(M \times T^{l}\right)$ is a norm limit of finite rank operators, i.e. is compact.

5.31 Proposition. If $\phi$ stands for the multiplication operator with the compactly supported $C^{1}$-function $\phi$ and $P$ for any first order equivariant differential operator, the commutator $[\phi, P]$ is compact as operator from $H^{1}(M) \rightarrow L^{2}(M)$.

The same applies if $P$ is replaced by $E$ or $P+E$ for any equivariant self-adjoint bounded operator $E$ which is a norm limit of operators with finite propagation. 
Proof. The commutator $[\phi, P]$ is a multiplication operator with a derivative of $\phi$, a compactly supported function. The latter ones are compact as maps from $H^{1}$ to $L^{2}$.

For the perturbation, we have to consider also the commutator $\phi E-E \phi$. By finite propagation, up to a norm-small error we can write $\phi E=\phi E \psi$ with a compactly supported $\psi$. Then we only need to use that $H^{1} \rightarrow L^{2} \stackrel{\phi}{\rightarrow} L^{2}$ and $H^{1} \rightarrow L^{2} \stackrel{\psi}{\rightarrow} L^{2}$ are compact by the Rellich lemma and that $E: L^{2} \rightarrow L^{2}$ is bounded.

5.32 Proposition. Let $D$ be an equivariant Dirac type operator on $M$ and $E: L^{2}(M) \rightarrow L^{2}(M)$ an $L^{2}$ bounded self-adjoint perturbation which is a norm limit of equivariant finite propagation operators such that $D+E$ becomes invertible. Assume that $\phi$ is a compactly supported $C^{1}$-function on $M \times N$. Then $|D+E|$, as map from $H^{1}(M \times N) \rightarrow L^{2}(M \times N)$, is a limit of equivariant bounded operators $F_{\epsilon}: H^{1}(M \times N) \rightarrow$ $L^{2}(M \times N)$ that are of finite propagation and such that $\left[F_{\epsilon}, \phi\right]: H^{1}(M \times N) \rightarrow L^{2}(M \times N)$ is compact.

Proof. We can reduce to $\phi=\alpha(x) \beta(y)$ with $\alpha: M \rightarrow \mathbb{C}, \beta: N \rightarrow \mathbb{C}$ compactly supported $C^{1}$-functions. We write $|D+E|: H^{1}(M \times N) \rightarrow L^{2}(M \times N)$ as

$$
H^{1}(M \times N) \stackrel{D+E}{\longrightarrow} L^{2}(M \times N)=L^{2}(M) \otimes L^{2}(N) \stackrel{(|D+E| /(D+E)) \otimes \mathrm{id}}{\longrightarrow} L^{2}(M) \otimes L^{2}(N)
$$

Using Lemma 2.26, write $|D+E| /(D+E)$ on $M$ as the limit of equivariant finite propagation pseudolocal operators $\kappa_{\epsilon}$ and consider $F_{\epsilon}$ given by the composition

$$
F_{\epsilon}: H^{1}(M \times N) \stackrel{D+E}{\longrightarrow} L^{2}(M \times N)=L^{2}(M) \otimes L^{2}(N) \stackrel{\kappa_{\epsilon} \otimes \mathrm{id}}{\longrightarrow} L^{2}(M) \otimes L^{2}(N)
$$

Observe that $F_{\epsilon}$ is of finite propagation as a bounded operator $H^{1}(M \times N) \rightarrow L^{2}(M \times N)$. We now show that it is also pseudolocal as a bounded operator $H^{1}(M \times N) \rightarrow L^{2}(M \times N)$. We have

$$
\left[\phi, F_{\epsilon}\right]=\left(\left[\alpha, \kappa_{\epsilon}\right]\right)(D+E) \otimes \beta+\kappa_{\epsilon}([\alpha,(D+E)]) \otimes \beta \text {. }
$$

Use here that $\beta$ commutes with all the other operators, which allows to split off the tensor factor $\beta$ in $\phi=\beta \alpha$ throughout. The second summand is compact by Proposition 5.31. The first summand is compact by Proposition 5.30

5.33 Proposition. Given a Dirac type operator $D$ as above, the operator $\left(1+D^{2}\right)^{-1}: L^{2} \rightarrow H^{2}$ is a norm limit of equivariant finite propagation operators $G_{\epsilon}: L^{2} \rightarrow H^{2}$ with the property that $\left[\phi, G_{\epsilon}\right]: L^{2} \rightarrow H^{2}$ is compact for any compactly supported smooth function on $M$.

Proof. We follow the idea of the proof of Proposition 4.19 in [16]. Consider $f(x):=\left(1+x^{2}\right)^{-1}$ and arrange that $f(x)=g_{\epsilon}(x)+h_{\epsilon}(x)$ where $g_{\epsilon}(x)$ has compactly supported Fourier transform and the Fourier transform of $h_{\epsilon}(x)$ together with its second derivative have small $L^{1}$-norm; use here that $\hat{f}(\xi)$, albeit non-smooth at 0 , is away from 0 smooth such that all derivatives are rapidly decreasing. Set $G_{\epsilon}:=g_{\epsilon}(D)$. Then $G_{\epsilon}$ has, by unit propagation of the wave operator, finite propagation, and

$$
\begin{aligned}
\left\|\left(1+D^{2}\right)^{-1}-G_{\epsilon}\right\|_{L^{2} \rightarrow H^{2}}=\left\|h_{\epsilon}(D)\right\|_{L^{2} \rightarrow H^{2}}=\left\|\left(1+D^{2}\right) h_{\epsilon}(D)\right\|_{L^{2} \rightarrow L^{2}} \leq|x| \mapsto & \left(1+x^{2}\right) h_{\epsilon}(x)_{\infty} \\
& \leq|\hat{h}|_{1}+\left|\hat{h}^{\prime \prime}\right|_{1}<<1
\end{aligned}
$$

The fact that $G_{\epsilon}$ is pseudolocal as a map $L^{2} \rightarrow H^{2}$ is proved by employing a doubling trick and (standard) microlocal techniques, as in [16].

A purely functional analytic argument, which applies to Lipschitz manifolds without a pseudodifferential calculus is given in [31]. 
5.34 Proposition. Let $D$ be a Dirac type operator on $M$ with equivariant self-adjoint bounded perturbation $E: L^{2}(M) \rightarrow L^{2}(M)$ as above such that $D+E$ is invertible and such that $E$ is the norm limit of equivariant finite propagation operators. Then $(D+E)^{-1}: L^{2} \rightarrow H^{1}$ is a norm limit of equivariant finite propagation operators $A_{\epsilon}: L^{2} \rightarrow H^{1}$ with the property that for any compactly supported smooth function $\phi$ the operator $\left[\phi, A_{\epsilon}\right]: L^{2} \rightarrow H^{1}$ is compact.

Proof. Write

$$
(D+E)^{-1}=(D+i)\left(1+D^{2}\right)^{-1}\left((D+E-i)(D+E)^{-1}-E(D+E)^{-1}\right) .
$$

Here, $(D+E-i)(D+E)^{-1}: L^{2} \rightarrow L^{2}$ is, by Lemma 2.26, an element in $D^{*}$, thus a norm limit of equivariant finite propagation pseudolocal operators $h_{\epsilon}$. Similarly $(D+E)^{-1}: L^{2} \rightarrow L^{2}$ and therefore $E(D+E)^{-1}: L^{2} \rightarrow$ $L^{2}$ are elements in $C^{*}$, and so the latter is a norm limit of equivariant finite propagation locally compact operators $\lambda_{\epsilon}$. Next, $D+i: H^{2} \rightarrow H^{1}$ is bounded with propagation 0 and has the property that $[\phi, D+$ $i]: H^{2} \rightarrow H^{1}$ is compact by the Rellich lemma. Finally, by Proposition 5.33 above, $\left(1+D^{2}\right)^{-1}: L^{2} \rightarrow H^{2}$ is the norm limit of equivariant finite propagation pseudolocal operators $G_{\epsilon}: L^{2} \rightarrow H^{2}$. Using the derivation property of $[\phi, \cdot]$ and approximating $(D+E)^{-1}$ by $A_{\epsilon}:=(D+i) G_{\epsilon}\left(h_{\epsilon}-\lambda_{\epsilon}\right)$ we easily conclude the proof.

5.35 Corollary. If $E: L^{2}(M) \rightarrow L^{2}(M)$ is a bounded self-adjoint equivariant operator and a norm limit of equivariant finite propagation operators and $D$ is a Dirac type operator on $M$ as above such that $D+E$ is invertible, then the operator $\frac{|D+E|+\partial_{t}}{D+E-\partial_{t}}: L^{2} \rightarrow L^{2}$ belongs to $D^{*}(M \times \mathbb{R})^{\Gamma}$.

Proof. We must prove that $\frac{|D+E|+\partial_{t}}{D+E-\partial_{t}}$ is the norm limit of a sequence of equivariant bounded operators $H_{\epsilon}: L^{2} \rightarrow L^{2}$ that are of finite propagation and pseudolocal. To check pseudolocality, by a density argument it suffices to consider the commutator with compactly supported smooth functions. We choose $N=\mathbb{R}$ in the previous propositions. We observe that $\left(D+E-\partial_{t}\right)$ is a summand of the perturbed Dirac type operator $\left(\begin{array}{cc}0 & D+E-\partial_{t} \\ D+E+\partial_{t} & 0\end{array}\right)$ on $M \times \mathbb{R}$; thus $\left(D+E-\partial_{t}\right)^{-1}: L^{2} \rightarrow H^{1}$ is a norm limit of equivariant operators $A_{\epsilon}: L^{2} \rightarrow H^{1}$ that are of finite propagation operators and commute up to compact operators with multiplication by compactly supported smooth functions. Here we have used Proposition 5.34 with $M \times \mathbb{R}$ instead of $M$. By Proposition 5.32 $|D+E|$, as map from $H^{1}(M \times \mathbb{R}) \rightarrow L^{2}(M \times \mathbb{R})$, is a limit of bounded equivariant operators $F_{\epsilon}: H^{1}(M \times \mathbb{R}) \rightarrow L^{2}(M \times \mathbb{R})$ that are of finite propagation and such that $\left[F_{\epsilon}, \phi\right]: H^{1}(M \times \mathbb{R}) \rightarrow L^{2}(M \times \mathbb{R})$ is compact. Finally, $\partial_{t}: H^{1} \rightarrow L^{2}$ has propagation zero and $\left[\phi, \partial_{t}\right]$ is clearly compact as a map $H^{1} \rightarrow L^{2}$ (Rellich Lemma). Summarizing, by writing

$$
\frac{|D+E|+\partial_{t}}{D+E-\partial_{t}}=\frac{|D+E|}{D+E-\partial_{t}}+\frac{\partial_{t}}{D+E-\partial_{t}}
$$

we see that $\frac{|D+E|+\partial_{t}}{D+E-\partial_{t}}$ is a sum of two elements in $D^{*}(M \times \mathbb{R})^{\Gamma}$ and it is therefore in $D^{*}(M \times \mathbb{R})^{\Gamma}$, as required.

\section{References}

[1] Robert Deeley and Magnus Goffeng. Realizing the analytic surgery group of Higson and Roe geometrically, part I: The geometric model. arXiv:1308.5990.

[2] Nigel Higson and John Roe. Mapping surgery to analysis. I. Analytic signatures. K-Theory, 33(4):277$299,2005$.

[3] Nigel Higson and John Roe. Mapping surgery to analysis. II. Geometric signatures. K-Theory, $33(4): 301-324,2005$.

[4] Nigel Higson and John Roe. Mapping surgery to analysis. III. Exact sequences. K-Theory, 33(4):325$346,2005$. 
[5] Michel Hilsum and Georges Skandalis. Invariance par homotopie de la signature à coefficients dans un fibré presque plat. J. Reine Angew. Math., 423:73-99, 1992.

[6] G. G. Kasparov. K-theory, group $C^{*}$-algebras, and higher signatures (conspectus). In Novikov conjectures, index theorems and rigidity, Vol. 1 (Oberwolfach, 1993), volume 226 of London Math. Soc. Lecture Note Ser., pages 101-146. Cambridge Univ. Press, Cambridge, 1995.

[7] Eric Leichtnam, John Lott, and Paolo Piazza. On the homotopy invariance of higher signatures for manifolds with boundary. J. Differential Geom., 54(3):561-633, 2000.

[8] Eric Leichtnam and Paolo Piazza. Spectral sections and higher Atiyah-Patodi-Singer index theory on Galois coverings. Geom. Funct. Anal., 8(1):17-58, 1998.

[9] Eric Leichtnam and Paolo Piazza. A higher Atiyah-Patodi-Singer index theorem for the signature operator on Galois coverings. Ann. Global Anal. Geom., 18(2):171-189, 2000.

[10] Eric Leichtnam and Paolo Piazza. Dirac index classes and the noncommutative spectral flow. J. Funct. Anal., 200(2):348-400, 2003.

[11] John Lott. Superconnections and higher index theory. Geom. Funct. Anal., 2(4):421-454, 1992.

[12] Wolfgang Lück. A basic introduction to surgery theory. In Topology of high-dimensional manifolds, No. 1, 2 (Trieste, 2001), volume 9 of ICTP Lect. Notes, pages 1-224. Abdus Salam Int. Cent. Theoret. Phys., Trieste, 2002.

[13] Richard B. Melrose and Paolo Piazza. Families of Dirac operators, boundaries and the $b$-calculus. J. Differential Geom., 46(1):99-180, 1997.

[14] Richard B. Melrose and Paolo Piazza. An index theorem for families of Dirac operators on odddimensional manifolds with boundary. J. Differential Geom., 46(2):287-334, 1997.

[15] Paolo Piazza and Thomas Schick. Bordism, rho-invariants and the Baum-Connes conjecture. J. Noncommut. Geom., 1(1):27-111, 2007.

[16] Paolo Piazza and Thomas Schick. Rho-classes, index theory and Stolz' positive scalar curvature sequence. J. Topol., 7(4):965-1004, 2014.

[17] Andrew Ranicki. Algebraic and geometric surgery. Oxford Mathematical Monographs. The Clarendon Press Oxford University Press, Oxford, 2002. Oxford Science Publications.

[18] John Roe. Comparing analytic assembly maps. Q. J. Math., 53(2):241-248, 2002.

[19] Jonathan Rosenberg and Shmuel Weinberger. The signature operator at 2. Topology, 45(1):47-63, 2006.

[20] Thomas Schick. $L^{2}$-index theorems, KK-theory, and connections. New York J. Math., 11:387-443 (electronic), 2005.

[21] Paul Siegel. Homological calculations with the analytic structure group. Ph.D. Thesis, Penn State University, 2012.

[22] Paul Siegel. The Mayer-Vietoris sequence for the analytic structure group. arXiv:1212.0241, 2012.

[23] Charlotte Wahl. Product formula for Atiyah-Patodi-Singer index classes and higher signatures. J. K-Theory, 6(2):285-337, 2010.

[24] Charlotte Wahl. The Atiyah-Patodi-Singer index theorem for Dirac operators over $C^{*}$-algebras. Asian J. Math., 17(2):265-319, 2013. 
[25] Charlotte Wahl. Higher $\rho$-invariants and the surgery structure set. J. Topol., 6(1):154-192, 2013.

[26] C. T. C. Wall. Surgery on compact manifolds, volume 69 of Mathematical Surveys and Monographs. American Mathematical Society, Providence, RI, second edition, 1999. Edited and with a foreword by A. A. Ranicki.

[27] N. E. Wegge-Olsen. K-theory and $C^{*}$-algebras. Oxford Science Publications. The Clarendon Press Oxford University Press, New York, 1993. A friendly approach.

[28] Zhizhang Xie and Guoliang Yu. Positive scalar curvature, higher rho invariants and localization algebras. Adv. Math., 262:823-866, 2014.

[29] Guoliang Yu. Localization algebras and the coarse Baum-Connes conjecture. K-Theory, 11(4):307-318, 1997.

[30] Rudolf Zeidler. Positive scalar curvature and product formulas for secondary index invariants. arXiv:1412.0685.

[31] Vito Felice Zenobi. Mapping the surgery sequence for topological manifolds to analysis. arXiv:1405.6323. 